\title{
Variations saisonnières de la composition et de la structure du pelage : exemple de la toison du lapin angora
}

\author{
J. ROUGEOT et R.-G. THEBAULT \\ I.N.R.A., Laboratoire des Pelages, Toisons et Fourrures \\ Centre de Recherches Zootechniques, 78350 Jouy-en-Josas
}

\section{Résumé}

La variation saisonnière de la production de poil du lapin angora - poids de la toison, longueurs et diamètres des trois catégories de poils, nombre de poils par groupe folliculaire - ont été analysés lors de chacun des épilages d'été, d'automne, d'hiver et de printemps, espacés de 98 jours, chez 22 lapines angora adultes. Les animaux étaient logés dans un bâtiment clos dont la température ne descendit jamais au-dessous de $10{ }^{\circ} \mathrm{C}$ et dont l'éclairage artificiel était réglé sur le photopériodisme naturel. Le régime alimentaire était constant.

Ces variations saisonnières des diverses caractéristiques de la toison se manifestent effectivement malgré la structure particulière du pelage angora, due à l'allongement de la durée de la croissance des poils, et en dépit de l'effet stimulant de l'épilation sur l'activité folliculaire. Elles sont régulées par le photopériodisme. Le poids des toisons récoltées est minimal en été (20 p. 100 de la production annuelle de $860 \mathrm{~g}$ en moyenne), maximal en automne $(26,8$ p. 100$)$ et en hiver $(27,6$ p. 100), intermédiaire au printemps $(25,5$ p. 100). Les composantes du poids varient toutes dans le même sens. Les longueurs diminuent en été dans des proportions d'autant plus élevées qu'il s'agit de catégories plus fines de poils : il en résulte une modification de la structure de la mèche où les barbes et surtout les duvets montent plus haut en automne-hiver qu'en été : 73,5 p. 100 contre 67,5 p. 100.

Les diamètres sont aussi plus faibles en été mais les écarts sont moins systématiques et moins prononcés. On suppose en outre que la variation du diamètre de la moelle peut atténuer l'effet de celles du diamètre des poils sur le poids de la toison.

Les analyses histologiques révèlent qu'une partie des follicules pileux disparaissent au printemps (-12,4 p. 100) par rapport à l'automne-hiver et surtout en été $(-28,2$ p. 100). Il s'agit uniquement de follicules pileux secondaires dérivés de follicules épidermiques. Ces follicules pileux se reconstituent par bourgeonnement en automne. On se trouve donc en présence d'un renouvellement folliculaire - et non d'une néogénèse - totalement différent du cycle folliculaire classique et qui a un effet majeur sur la compacité du pelage.

Ces observations permettent de préciser la notion de follicule pileux composé, notamment en lui attribuant deux fonctions de première importance pour le pelage : le développement du sous-poil chez le jeune mammifère et l'adaptation du pelage de l'adulte aux changements climatiques saisonniers. Ces résultats sont comparés à des observations faites par d'autres auteurs sur le lapin angora et d'autres espèces. 


\section{Introduction}

Le pelage de la plupart des mammifères, en particulier de ceux qui vivent sous les latitudes tempérées et sub-polaires, subit des mues saisonnières réglées par le photopériodisme (Bissonnette, 1935, sur le furet; Bissonnette \& Wilson, 1939, sur le vison; Bissonnette \& Bailey, 1944, sur la belette; Bassett, Pearson \& Wilke, 1944, sur le renard; Belyaev \& UtKIn, 1949, sur le renard; Hammond, 1951, 1952, sur le furet et le vison; Yeates 1955, sur les bovins ; Rovgeot, 1957, 1961, 1972, sur les ovins; Harvey \& MacFarlane, 1958, sur le furet; Al-Khateeb \& Johnson, 1971 b, sur le Campagnol ; Ryder \& LinCOLN, 1976, sur le mouton; KAY \& RYder, 1978, sur le cerf; Rougeot, Thebault \& Allain 1982, sur le mouton et le vison).

Ces mues progressent sur le corps selon un gradient qui leur est caractéristique et fournissent, l'une au printemps, le pelage maigre et court de l'été, l'autre, à l'automne, le pelage dru et long de l'hiver. A ces modifications de composition et de structure s'ajoutent souvent des changements de nuances, sinon de couleur, le pelage d'été étant généralement plus foncé que celui d'hiver; ce dernier peut même devenir entièrement blanc (hermine, lièvre variable : Rothschild, 1942, 1944 ; Hewson, 1963 ; Watson, 1963 ; Borowski, 1964 ; Flux, 1970). Ainsi le rôle des mues ne consiste pas seulement à renouveler un pelage usé, selon un synchronisme plus ou moins accentué, mais aussi à fournir un pelage adapté aux changements climatiques saisonniers (EBLING \& HALE, 1970 ; Ling, 1970 ; Johnson, 1977 b ; Rougeot, 1981 ; Rougeot, Thebault \& All.ain, 1982).

Par contre chez des espèces non saisonnières comme le rat ou la souris de laboratoire, le renouvellement du pelage s'étale sur toute l'année, les phases d'activité du follicule pileux (anagène) s'étendant sur une vingtaine de jours contre 5 à 10 jours pour celle de repos (télogène) (Dry, 1926; Butcher, 1934, 1951; Chase, 1954 ; Priestley, 1966). Chez le jeune animal, l'activité folliculaire progresse sur le corps par vagues successives selon des gradients définis. L'ordre de ces vagues se détruit avec l'âge, si bien que chez l'adulte le renouvellement des poils procède par plages irrégulières. C'est ce qui explique la nécessité de remettre en synchronisme les follicules pileux par l'épilation qui induit un nouvel anagène (Collins, 1918; David, 1934; Chase, 1946; Rothman, 1954) afin d'étudier sans interaction avec les cycles folliculaires naturels, l'effet de certains agents (rayonnement, hormones, nutrition, produits chimiques, etc.).

Le pelage du lapin domestique se rapproche de ce mode de fonctionnement, avec cependant une influence marquée de la saison chez l'adulte, ce qui conduit à un pelage stable en hiver (Rovgeot \& Thebault, données non publiées).

Chez le lapin angora, le remplacement des poils se complique avec l'interférence du prolongement de l'anagène qui dure au moins 13-16 semaines au lieu de 5 en moyenne chez le lapin ordinaire à pelage court (RougEor, 1974). On récolte donc le pelage, en fin de croissance, au maximum de longueur des poils, avant que ceux-ci ne tombent et feutrent la toison, le télogène ne durant que quelques jours. Cette récolte se fait soit par tonte, soit, comme le pratiquent les éleveurs français, par épilation. Cette épilation facilitée par la présence d'une forte proportion de follicules pileux en télogène, induit la croissance en synchronisme d'un nouveau pelage, parfaitement structuré, tous les poils revêtant l'intégralité de leur forme. Par contre, à la suite d'une tonte, 
la croissance du pelage se poursuit normalement avec une proportion importante de poils coupés, ce qui conduit à une toison dépourvue de structure.

Mais dans un cas comme dans l'autre, les récoltes d'automne et d'hiver fournissent toujours plus de poils que celles de l'été (25 à 30 p. 100) : cet effet saisonnier, explicable dans le cas de la tonte, paraît en contradiction avec ce que nous savons de l'action de l'épilation qui est supposée remettre en activité la totalité des follicules pileux.

Nous sommes donc amenés à préciser dans cette étude l'amplitude des variations saisonnières du poids de poils récoltés par épilation chez le lapin angora en analysant la part revenant à chaque paramètre de cette production : nombre de poils implantés dans la peau et dimensions des poils, c'est-à-dire diamètre et longueur.

\section{Matériel et méthodes}

\section{A. Animaux}

Les observations ont été poursuivies pendant une année sur 22 lapines angora âgées de 10 à 12 mois au début de l'expérience. Nous avons choisi des femelles car ce sont elles qui constituent dans les élevages le troupeau de production, étant donné qu'elles produisent un pelage plus abondant que les mâles ( 35 p. 100 en plus) et comprenant des poils plus longs et plus fins.

En tant que productrices de poil, ces femelles étaient soustraites à toute activité de reproduction. Elles étaient logées individuellement dans des clapiers en ciment, sur une litière de paille. En assurant ainsi leur confort, on obtient une croissance maximale des poils en évitant notamment les chutes accidentelles de production qui se produisent lorsque les animaux sont maintenus sur grillage. Les escarres qui apparaissent avec ce matériel à la face inférieure des pattes provoquent parfois une diminution de la production de poil atteignant 25 à 50 p. 100 (Rougeot, Colin \& Thebault, 1980). Ces clapiers étaient disposés dans un local clos, l'éclairage étant fourni par des bulbes à décharge donnant un éclairement d'environ 500 lux au niveau des portes grillagées des cages. La durée journalière de l'éclairage était réglée par une cellule photoélectrique située à l'extérieur du bâtiment afin de suivre la variation normale du jour sous la latitude $45^{\circ}$ nord.

Les lapines étaient nourries exclusivement avec un aliment complet aggloméré dont la teneur en protéines brutes s'élevait à 17 p. 100 (soit 12,3 p. 100 digestibles; LEBAs, 1975 ), celle en matières grasses à 3,2 p. 100 (2,5 p. 100 digestibles) et celle de cellulose à 16 p. 100 (4 p. 100 digestibles). L'énergie digestible fournie par cet aliment se montait à $2700 \mathrm{kcal} / \mathrm{kg}$ (LEBAS, 1975). Cet aliment granulé était distribué chaque jour à raison de $180 \mathrm{~g}$ par lapine dans des trémies fixées sur la porte afin d'éviter toute perte et faciliter le contrôle. En outre, chaque vendredi, on apportait entre 250 et $300 \mathrm{~g}$ de paille propre sur la litière afin, non seulement d'assurer la propreté de celle-ci, mais aussi de fournir un lest alimentaire supplémentaire, les besoins individuels étant très variables en cette matière (Rougeot, Colin \& ThebaulT, 1980). L'abreuvement était assuré en permanence par des abreuvoirs automatiques. 


\section{B. Mesures des caractéristiques de la toison récoltée}

\section{Récoltes de poil}

Les récoltes furent effectuées, par épilation, tous les 98 jours, la maturité du pelage étant atteinte au terme de ce temps de croissance. Cependant, pour faciliter l'arrachage des poils et atténuer le choc d'épilation, on provoqua une brève interruption de l'activité des follicules pileux, par l'administration orale de $70 \mathrm{mg} / \mathrm{kg}$ vif de cyclophosphamide incorporé à l'aliment granulé (Asta Werke, Brackwede, R.F.A.) 6 jours avant la date prévue de la récolte (Rougeot \& Thebault, 1970, 1976). Les 4 récoltes de l'année furent réparties en septembre pour les toisons d'été (croissance de juin à septembre), en décembre pour les toisons d'automne (croissance de septembre à décembre), en mars pour les toisons d'hiver (croissance de décembre à mars) et en juin pour les toisons de printemps (croissance de mars à juin).

\section{Poids}

A chaque récolte, la toison, à l'exclusion des poils de tête et de l'extrémité des pattes, a été pesée au gramme près.

\section{Compacité de la toison : le groupe folliculaire du lapin}

Les mesures de surface de peau sur l'animal sont entâchées derreurs en raison de l'élasticité et de la plasticité du tégument. C'est pourquoi, lorsqu'on veut suivre sur un même individu la variation du nombre de poils implantés dans la peau, on s'adresse aux caractéristiques du groupe folliculaire qui sont indépendantes de l'extension ou du rétrécissement de la peau. Le groupe folliculaire type du lapin (fig. 1) se compose : a) d'un follicule pileux primaire central (PC), seul muni d'un muscle arrecteur et produisant le jarre, poil recteur, de fort diamètre jusqu’à la base (fig. 2); b) de 4 follicules pileux primaires latéraux (PL, deux d'ordre 1 et deux d'ordre 2) fournissant les barbes, poils tecteurs dont les têtes renflées comme celles des jarres s'inclinent sur un corps relativement fin. Les jarres et les barbes sont des poils de garde; c) de follicules pileux secondaires (S) assemblés en faisceaux accompagnant chaque barbe ; ils produisent les courts et fins duvets qui constituent le sous-poil dont la fonction est l'isolation thermique. Le PC est un élément permanent du groupe folliculaire facilement reconnaissable par son isolement, sa taille et la présence du muscle arrecteur. Par contre, les barbes, inclues dans le faisceau du duvet ne sont pas toujours repérables, surtout quand leur diamètre se réduit en fin de croissance. Aussi définit-on la densité d'implantation

des follicules pileux, chez le lapin, par le rapport $\frac{\mathrm{PL}+\mathrm{S}}{\mathrm{PC}}$ de la somme des PL

et des $\mathrm{S}$ confondus sur le nombre de PC (MehNer \& Koetter, 1965; RusaouËN, 1966 ; Thebault, 1977) : on obtient ainsi une valeur correspondant au nombre moyen de follicules pileux composant le groupe folliculaire, nombre lui-même lié à la compacité de la toison. Le dénombrement des follicules pileux est effectué sur coupes histologiques. Dans cette étude, les prélèvements de peau ont été opérés sur le dos, 5 semaines après l'épilation, au maximum de croissance des poils (Rougeot, 1974) à laide d'un emporte-pièce circulaire de $33 \mathrm{~mm}^{2}$; fixation au Bouin alcoolique pendant 48 heures; après inclusion à la paraffine, coupes à $8 \mathrm{ltm}$ d'épaisseur, parallèlement à l'épiderme ; coloration rapide au trichrome Roan (Rouge solide, Orange G, Bleu d'ani- 
line, Rougeot, 1972) qui permet de distinguer nettement les follicules pileux, en section droite, à la projection microscopique au grossissement 70 sur écran en verre dépoli; on projette la coupe entière pour éviter toute erreur dans le comptage. Ces observations ont également fourni les proportions de $\mathrm{PC}$ en anagène et en télogène.

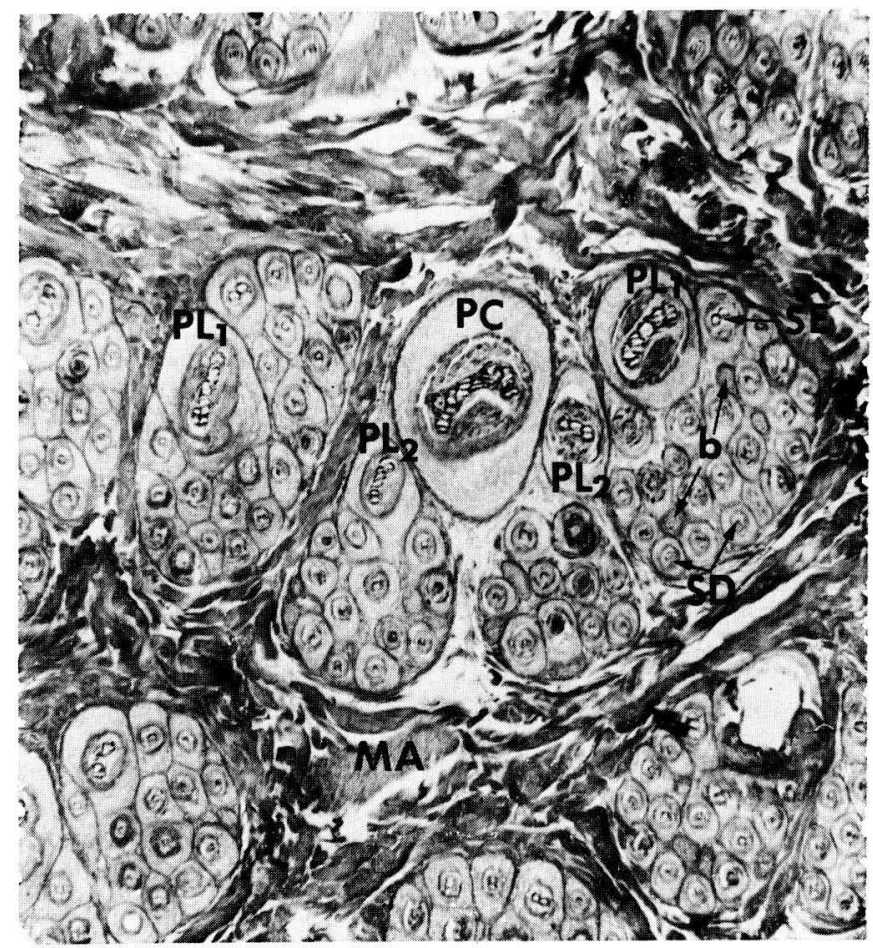

FIG. 1

Le groupe folliculaire type du lapin angora.

Typical hair follicle group of the angora rabbit.

PC : Follicule primaire central producteur de jarre; poil recteur. Central primary follicle producing bristle; rector hair.

PL : Follicules primaires latéraux producteurs de barbes; poils tecteurs. PL $\mathbf{1}$ : de premier ordre, PL. : de second ordre.

Lateral primary follicles producing awn; tector hair. $P L_{t}$ : of first order, $P L_{\mathbf{z}}$ : of second order.

S : Follicules secondaires, producteurs de duvets. SE : épidermiques, SD : dérivés. Secondary follicles producing down. SE : epidermal, SD : derived.

b : Bourgeons pileux.

Hair germ.

MA : Muscle arrecteur.

Arrector muscle. 


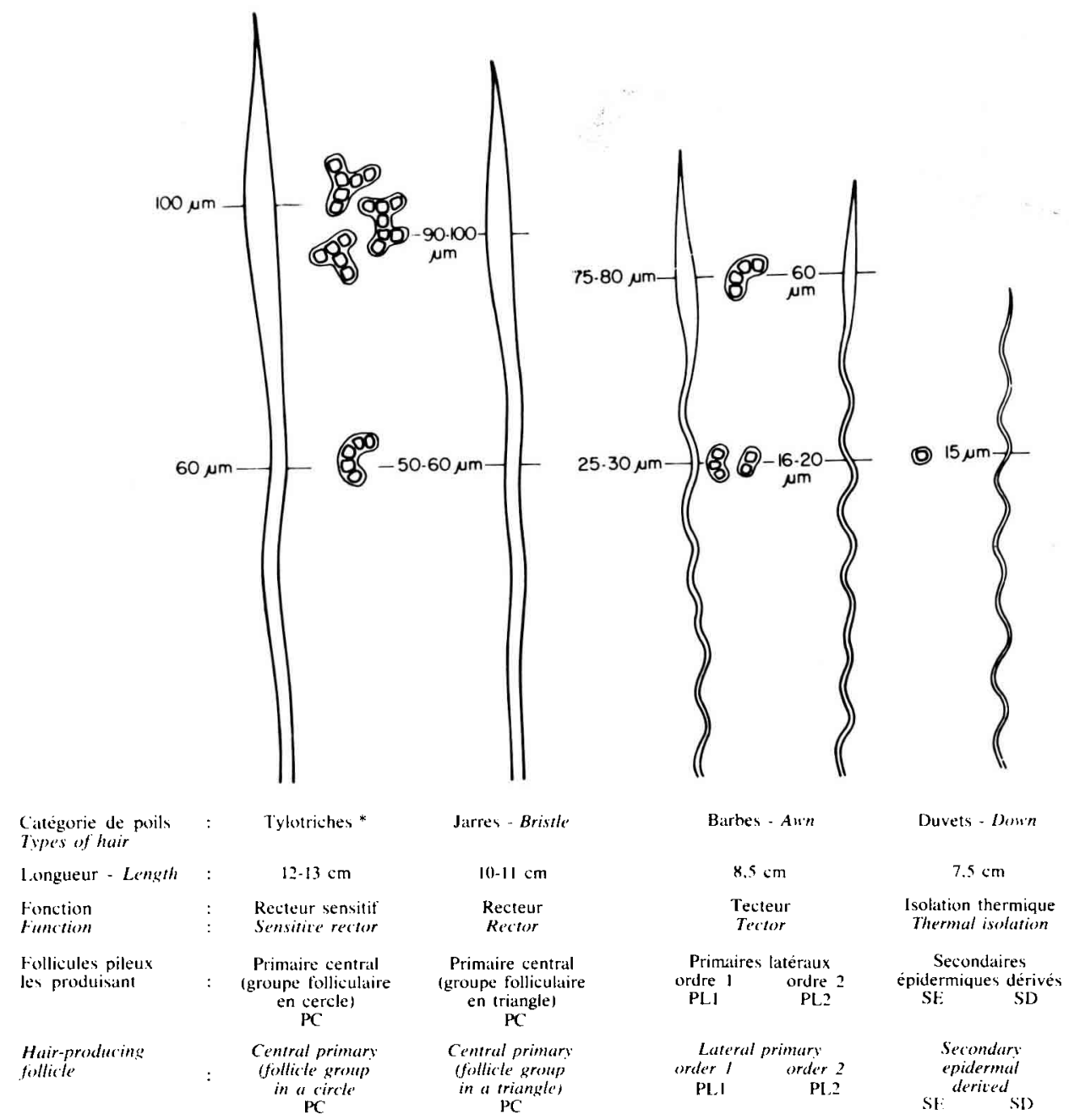

FIG. 2

Les différentes catégories de poils du Lapin angora : vue de profil de la tige du poil, sections droites de la tête et du corps avec leurs diamètres moyens.

Different types of Angora rabbit hair: profile hair shaft. sections of the shield and basal shaft with their mean diameters.

* On dénombre, en moyenne, 3 tylotriches (Stralle, 1960) par $\mathrm{cm}^{2}$ de peau. Leur morphologie, à part une longueur et un diamètre légèrement supérieurs, est identique à celle des jarres. Dans nos mesures, nous avons donc groupé ensemble tous les poils recteurs, tylotriches et jarres. There are 3 tylotriches per $\mathrm{cm}^{2}$ of skin. Their morphology is the same as that of bristles, except that they are slighty longer and thicker. Our measurements group together all rector hair, tylotriches and bristles. 


\section{Longueurs}

Les mesures ont été effectuées sur des mèches prélevées par rasage sur le dos des lapines, juste avant de procéder à la récolte. La mèche d'un pelage d'épilation étant parfaitement structurée, les sommets des 3 catégories de poils se situent à des niveaux nettement séparés, ce qui permet leur tri mécanique à l'aide de l'appareil à pinces décrit par Thebault (1977). Les jarres sont extraits les premiers et, comme leurs longueurs sont assez inégales, on les mesure individuellement en les étendant le long d'une règle graduée; 90 jarres par mèche ont été ainsi mesurés. Quant aux barbes et aux duvets, dont les bases sont coincées en place dans l'appareil à pinces et qui ont été parallélisés et étirés au peigne, ils présentent des sommets alignés sur une droite parallèle à celle des racines : les hauteurs des sous-mèches de barbes et de duvets, mesurées au $\mathrm{mm}$ près, fournissent une estimation de leur longueur moyenne.

\section{Mesure des diamètres}

Les diamètres ont été mesurés suivant la norme IWTO 8-61 (F) sur la mesure microscopique du diamètre de la laine (Fédération Internationale Lainière, 1966). Les diamètres sont mesurés par projection au grossissement 500 (Lanamètre Reichert) selon le profil, sur des fragments de poils (têtes exclues) obtenus au fibrotome ITF de Lhomargy (JaCQuemarT \& MonrocQ, 1965), après conditionnement des poils à $20^{\circ} \mathrm{C}$ et 65 p. 100 d'humidité relative. Trente diamètres de chaque catégorie de poils ont été mesurés par mèche.

\section{Méthodologie statistique}

L'exploitation statistique des résultats, en ce qui concerne la variation de la production de poil, la variation de la longueur des poils et la densité folliculaire dans la peau a été effectuée par la méthode de comparaison de moyennes de Student, test « $t$ » par paires ou par couples. En ce qui concerne la variation des diamètres des fibres, nous avons utilisé le test « $t »$ relatif aux échantillons indépendants. Dans les deux cas nous avons fixé les seuils de signification à $P=0,05$ pour significatif et à $P=0,01$ pour hautement significatif.

\section{Résultats}

\section{A. Poids saisonniers des toisons (tabl. 1)}

La production moyenne annuelle des lapines qui est de $860 \mathrm{~g}$ manifeste des variations étroitement liées à la saison.

Les toisons d'été représentent 20 p. 100 de la récolte annuelle : elles sont significativement plus légères que celles produites aux trois autres saisons. La différence de leur poids est même hautement significative par rapport à ceux des toisons d'automne (26,8 p. 100 de la production annuelle) et d'hiver $(27,6$ p. 100$)$ qui sont équivalentes et atteignent les valeurs les plus élevées. Les poids des toisons de printemps se trouvent être intermédiaires entre ceux des toisons d'été d'autre part, les différences étant toutes significatives; avec 25,5 p. 100 , la récolte de printemps se situe juste dans la moyenne de la production annuelle. 


\section{Tableau 1}

Moyennes des poids des toisons des 22 lapines angora, selon les saisons.

Fleece weight means of 22 angora does according to season.

\begin{tabular}{|c|c|c|c|c|c|}
\hline $\begin{array}{l}\text { Type de toison } \\
\text { Type of fleece }\end{array}$ & $\begin{array}{c}\text { Eté } \\
\text { Summer }\end{array}$ & $\begin{array}{l}\text { Automne } \\
\text { Autumn }\end{array}$ & $\begin{array}{l}\text { Hiver } \\
\text { Winter }\end{array}$ & $\begin{array}{l}\text { Printemps } \\
\text { Spring }\end{array}$ & $\begin{array}{c}\text { Moyenne } \\
\text { annuelle } \\
\text { Yearly } \\
\text { mean }\end{array}$ \\
\hline $\begin{array}{c}\text { Période de croissance } \\
\text { des poils (98 jours) } \\
\text { Hair growth period } \\
(98 \text { days) }\end{array}$ & $\begin{array}{l}\text { juin-sept. } \\
\text { June-Sept. }\end{array}$ & $\begin{array}{l}\text { sept.-déc. } \\
\text { Sept.-Dec. }\end{array}$ & $\begin{array}{c}\text { déc.-mars } \\
\text { Dec.-March }\end{array}$ & $\begin{array}{c}\text { mars-juin } \\
\text { March-June }\end{array}$ & \\
\hline$\overline{\mathrm{x}} \pm \mathrm{s}_{\overline{\mathrm{x}}}$ (en $\left.\mathrm{g}\right) \ldots \ldots$ & $172,4 \pm 5,6$ & $230,4 \pm 4,8$ & $237,4 \pm 6,0$ & $2 \cdot 18,6 \pm 5,5$ & 858,8 \\
\hline$\ldots \ldots \ldots \ldots \ldots$ & 20,1 & 26,8 & 27,6 & 25,5 & 100 \\
\hline
\end{tabular}

B. Variation des longueurs des trois catégories de poils selon la saison

(tabl. 2)

Quelle que soit la catégorie de poils, ce sont ceux qui poussent en été qui sont les plus courts et cela de façon significative par rapport à ceux produits à chacune des autres saisons. La longueur des poils d'été est inférieure d'environ $0,75 \mathrm{~cm}$ à la longueur moyenne des 4 saisons, soit de 7,2 p. 100 pour les jarres, de 10,4 p. 100 pour les barbes et de 11,3 p. 100 pour les duvets. Par contre en automne, hiver et printemps les longueurs des 3 catégories de poils sont toutes supérieures à cette longueur moyenne annuelle, mais avec des écarts plus atténués que pour les poils d'été.

Les longueurs maximales sont atteintes en hiver pour les barbes et les duvets : elles sont significativement supérieures aux longueurs d'automne et do printemps. Pour les jarres, c'est en automne que la longueur maximale est atteinte avec une différence significative par rapport à celle du printemps mais pas avec celle d'hiver. Les différences entre longueurs intermédiaires, hiver-printemps pour les jarres, automne-printemps pour les barbes et les duvets ne sont pas significatives.

L'écart maximal des longueurs est donc situé entre les poils d'été et ceux d'hiver et il s'accentue lorsqu'on passe des catégories les plus grossières aux plus fines : il est de 9,9 p. 100 pour les jarres (en prenant pour longueur maximale la moyenne des longueurs d'automne et d'hiver), de 15,2 p. 100 pour les barbes et de 16,8 p. 100 pour les duvets, soit exprimé en rapport de longueurs, respectivement $1,11,1,18$ et 1,20.

Il résulte de cette variation de longueurs, plus ample chez les barbes et les duvets par rapport aux jarres, une modification de la structure sur la hauteur de la mèche suivant les saisons : les barbes occupent 83,4 p. 100 de la hauteur en hiver contre 78,5 p. 100 en été, l'écart s'accentuant avec les duvets qui montent à 73,5 p. 100 de la hauteur dans la mèche d'hiver contre 67,5 p. 100 dans celle d'été. Aux saisons 
intermédiaires, la structure des mèches dautomne se rapproche de celle d'été (barbes 79,9 p. 100 de la hauteur; duvets 63,7 p. 100) et celle des mèches de printemps de celle d'hiver (barbes 82,7 p. 100 de la hauteur; duvets 72,1 p. 100 ).

\section{TABleaU 2}

Longueurs moyennes des trois catégories de poils des mèches du dos de 22 lapines angora selon la saison.

Mean length of three types of hair from the back staples of 22 angora does according to season.

\begin{tabular}{|c|c|c|c|c|c|}
\hline \multirow{2}{*}{$\begin{array}{l}\text { Catégories de poils } \\
\text { Types of hair }\end{array}$} & \multicolumn{4}{|c|}{ Saisons de croissance de la toison - Hair growth seasons } & \multirow{2}{*}{$\begin{array}{l}\text { Moyenne } \\
\text { annuelle } \\
\text { Ycarly } \\
\text { mean }\end{array}$} \\
\hline & $\begin{array}{c}\text { Eté } \\
\text { Summor }\end{array}$ & $\begin{array}{l}\text { Automne } \\
\text { Autumn }\end{array}$ & $\begin{array}{l}\text { Hiver } \\
\text { Winter }\end{array}$ & $\begin{array}{l}\text { Printemps } \\
\text { Spring }\end{array}$ & \\
\hline $\begin{array}{c}\text { Jarres - Bristle } \\
\overline{\mathrm{x}}+\mathrm{s}_{\mathrm{x}}(\mathrm{en} \mathrm{cm}) \ldots .\end{array}$ & $9,26 \pm 0,15$ & $10,31 \pm 0,12$ & $10,24 \pm 0,09$ & $10,11 \pm 0,11$ & 9,98 \\
\hline $\begin{array}{l}\text { Ecar1/moy. annuclle } \\
- \text { en } \operatorname{cm} \ldots \ldots \ldots \\
- \text { en } \% \ldots \ldots \ldots\end{array}$ & $\begin{array}{l}-0,72 \\
-7,2 \\
\end{array}$ & $\begin{array}{l}+0,33 \\
+3,3 \\
\end{array}$ & $\begin{array}{l}+0,26 \\
+2,6 \\
\end{array}$ & $\begin{array}{l}+0,13 \\
+1,3\end{array}$ & \\
\hline $\begin{aligned} & \text { Barbes }-A w n \\
& \bar{x}+s_{i}(\text { en } \mathrm{cm}) \ldots\end{aligned}$ & $7,27 \pm 0,08$ & $8,24 \pm 0,12$ & $8,57 \pm 0,09$ & $8,36 \pm 0,10$ & 8,11 \\
\hline $\begin{array}{l}\text { Ecart/moy. annuelle } \\
- \text { en } \mathrm{cm} \ldots \ldots \ldots \\
\text { - en } \% \ldots \ldots \ldots\end{array}$ & $\begin{array}{c}-0,84 \\
-10,4\end{array}$ & $\begin{array}{l}+0,13 \\
+1,6\end{array}$ & $\begin{array}{l}+0,46 \\
+\quad 5,7\end{array}$ & $\begin{array}{r}+0,25 \\
+\quad 3,1 \\
\end{array}$ & \\
\hline $\begin{array}{c}\text { Duvets - Down } \\
\overline{\mathrm{x}}+\mathrm{s}_{\overline{\mathrm{x}}}(\mathrm{en} \mathrm{cm}) \ldots \ldots\end{array}$ & $6,26 \pm 0,10$ & $7,19 \pm 0,08$ & $7,51 \pm 0,09$ & $7,29 \pm 0,08$ & 7,06 \\
\hline $\begin{array}{l}\text { Ecart/moy. annuelle } \\
- \text { en } \mathrm{cm} \ldots \ldots \ldots \ldots \\
- \text { en } \% \ldots \ldots \ldots \ldots\end{array}$ & $\begin{array}{l}-0,80 \\
-11,3\end{array}$ & $\begin{array}{l}+0,13 \\
+1,8\end{array}$ & $\begin{array}{l}+0,46 \\
+6,4\end{array}$ & $\begin{array}{l}+0,23 \\
+3,3\end{array}$ & \\
\hline
\end{tabular}

Ecart/moy, ann. : difference from yearly mean.

C. Variation des diamètres des trois catégories de poils selon la saison

En été, les poils de chacune des trois catégories sont toujours plus fins qu'aux autres saisons (tabl. 3). Les différences des diamètres d'été par rapport à ceux des trois autres saisons sont toutes significatives, sauf pour les barbes entre été et automne. Les diamètres les plus forts se situent soit au printemps, soit en hiver, les valeurs d'automne pour les jarres et duvets s'en rapprochant toutefois plus ou moins. Ainsi pour les jarres, c'est le diamètre du printemps qui est le plus élevé, se distinguant à peine de celui de lautomne. Ils se montrent ainsi tous les deux significativement 
supérieurs à celui de l'hiver. Pour les barbes, c'est également le diamètre du printemps qui est le plus fort ; il est significativement plus élevé que celui d'hiver et par conséquent que celui d'automne voisin de celui de l'été ; enfin, pour les duvets, c'est le diamètre d'hiver qui est le plus épais, mais sans différence significative par rapport à ceux du printemps et de l'automne.

\section{TABLEAU 3}

Diamètres moyens des trois catégories de poils du dos de 22 lapines angora selon la saison.

Mean diameter of three types of hair from the back of 22 angora does according to season.

\begin{tabular}{|c|c|c|c|c|c|}
\hline \multirow{2}{*}{$\begin{array}{c}\text { Catégories de poils } \\
\text { Types of hair }\end{array}$} & \multicolumn{4}{|c|}{ Saisons de croissance de la toison - Hair growth seasons } & \multirow{2}{*}{$\begin{array}{c}\text { Moyenne } \\
\text { annuelle } \\
\text { Yearly } \\
\text { mean }\end{array}$} \\
\hline & $\begin{array}{c}\text { Eté } \\
\text { Summer }\end{array}$ & $\begin{array}{l}\text { Automne } \\
\text { Autumn }\end{array}$ & $\begin{array}{l}\text { Hiver } \\
\text { Winter }\end{array}$ & $\begin{array}{l}\text { Printemps } \\
\text { Spring }\end{array}$ & \\
\hline $\begin{array}{c}\text { Jarres - Bristle } \\
\overrightarrow{\mathbf{x}}+\mathrm{s}_{\overline{\mathbf{x}}}(\text { en } \mu \mathrm{m}) \ldots \ldots\end{array}$ & $58,06 \pm 0,38$ & $60,00 \pm 0,34$ & $58,92 \pm 0,36$ & $60,05 \pm 0,38$ & 59,26 \\
\hline $\begin{array}{l}\text { Ecart } / \text { moy. annuelle } \\
- \text { en } \mu \mathrm{m} \ldots \ldots \ldots \\
- \text { en } \% \quad \ldots \ldots \ldots \ldots\end{array}$ & $\begin{array}{l}1,20 \\
-2,0 \\
\end{array}$ & $\begin{array}{l}+0,74 \\
+1,2 \\
\end{array}$ & $\begin{array}{l}-0,34 \\
-0,6 \\
\end{array}$ & $\begin{array}{l}+0,74 \\
+1,3 \\
\end{array}$ & \\
\hline $\begin{array}{c}\text { Barbes - Awn } \\
\overrightarrow{\mathrm{x}}+\mathrm{s}_{\overline{\mathrm{x}}}(\mathrm{en} \mu \mathrm{m}) \ldots \ldots\end{array}$ & $29,18 \pm 0,22$ & $29,26 \pm 0,22$ & $30,24 \pm 0,222$ & $30,80 \pm 0,12$ & 29,87 \\
\hline $\begin{array}{l}\text { Ecart/moy. annuelle } \\
- \text { en } \mu \mathrm{m} \ldots \ldots \ldots \ldots \\
\text { en } \% \quad \ldots \ldots \ldots \ldots\end{array}$ & $\begin{array}{l}-0,79 \\
-2,3\end{array}$ & $\begin{array}{l}-0,61 \\
-2,0\end{array}$ & $\begin{array}{l}+0,37 \\
+1,2 \\
\end{array}$ & $\begin{array}{l}+0,93 \\
+\quad 3,1\end{array}$ & \\
\hline $\begin{array}{c}\text { Duvets - Down } \\
\overline{\mathrm{x}}+\mathrm{s}_{\overline{\mathrm{x}}}(\mathrm{en} \mu \mathrm{m}) \ldots\end{array}$ & $16,06 \pm 0,12$ & $16,88 \pm 0,12$ & $17,06 \pm 0,22$ & $16,92 \pm 0,12$ & 16,73 \\
\hline $\begin{array}{l}\text { Ecart/moy. annuelle } \\
- \text { en } \mu \mathrm{m} \ldots \ldots \ldots \\
- \text { en } \% \ldots \ldots \ldots\end{array}$ & $\begin{array}{l}-0,67 \\
-4,0\end{array}$ & $\begin{array}{l}+0,15 \\
-0,9\end{array}$ & $\begin{array}{l}+0,33 \\
+2,0\end{array}$ & $\begin{array}{l}+0,13 \\
+1,1\end{array}$ & \\
\hline
\end{tabular}

Comme pour les longueurs, la diminution saisonnière du diamètre en été s'accentue lorsqu'on passe de la catégorie des poils les plus grossiers à la catégorie des poils les plus fins. Toutefois, les écarts entre les diamètres d'été et les diamètres les plus forts de l'hiver ou du printemps paraissent moins prononcés : 3 p. 100 pour les jarres, 5 p. 100 pour les barbes, 6 p. 100 pour les duvets. Cette impression s'atténue si l'on s'adresse aux sections droites qui traduisent mieux la réalité de la production et dont on obtient une approximation en les supposant circulaires : on obtient alors respectivement 6,5 p. $100,10,3$ p. 100 et 11,3 p. 100 . 


\section{TableaU 4}

Variation saisonnière dans la population des follicules pileux de 22 lapines angora.

Seasonal variation in the hair follicle population of 22 angora does.

\begin{tabular}{|c|c|c|c|c|}
\hline & \multicolumn{4}{|c|}{ Saisons de croissance de la toison - Hair growth seasons } \\
\hline & $\begin{array}{c}\text { Eté } \\
\text { Summer }\end{array}$ & $\begin{array}{l}\text { Automne } \\
\text { Autumn }\end{array}$ & $\begin{array}{l}\text { Hiver } \\
\text { Winter }\end{array}$ & $\begin{array}{l}\text { Printemps } \\
\text { Spring }\end{array}$ \\
\hline $\begin{array}{c}\% \text { PC en anagène } \\
\% \text { PC in anagen } \\
\left(\overline{\mathrm{x}}+\mathrm{s}_{\overline{\mathrm{x}}}\right)\end{array}$ & $84 \pm 3$ & $95 \pm 2$ & $90 \pm 2$ & $92 \pm 1$ \\
\hline$\frac{\mathrm{PL}+\mathrm{S}}{\left(\overline{\mathrm{PC}}+\mathrm{s}_{\overline{\mathrm{x}}}\right)}$ & $37,0 \pm 1,8$ & $51,5 \pm 1,5$ & $49,5 \pm 1,7$ & $45,1 \pm 1,7$ \\
\hline $\begin{array}{l}\text { Ecart/automne : } \\
\text { Difference/autumn } \\
\text { - absolu ......... } \\
\text { absolute value } \\
\text { - en } \% \text {........ } \\
\text { in } \%\end{array}$ & $\begin{array}{l}-14,50 \\
-28,2\end{array}$ & - & $\begin{array}{l}-1,96 \\
-3,8\end{array}$ & $\begin{array}{l}-6,39 \\
-12,4\end{array}$ \\
\hline
\end{tabular}

PC : Nombre de follicules pileux primaires centraux.

Number of central primary follicles.

PL : Nombre de follicules pileux primaires latéraux.

Number of lateral primary follicles.

$\mathrm{S}$ : Nombre de follicules pileux secondaires.

Number of secondary follicles.

D. Variation saisonnière dans la population des follicules pileux

1. Vérification de la permanence des follicules pileux primaires centraux $(P C)$

Cinq semaines après l'épilation, on observe un certain nombre de PC en télogène, malgré l'effet inducteur de l'anagène de cette opération. Mais la grande majorité des PC demeure normalement en anagène en présentant de nettes fluctuations saisonnières : c'est en été que leur proportion est la plus faible, tombant à $84 \pm 3$ p. 100 , ce qui est significativement inférieur aux autres saisons; en automne, le taux remonte à 95 p. 100 pour redescendre en hiver et au printemps où il atteint des niveaux identiques : $90 \pm 2$ p. 100 et $92 \pm 1$ p. 100 respectivement, lesquels sont significatviement différents de celui de l'automne.

De toute façon, l'emplacement des PC étant facilement et indubitablement repérable dans les groupes folliculaires, nous avons toujours constaté leur présence, soit à l'état d'anagène pour la majorité d'entre eux, soit à l'état de télogène : les PC sont donc bien des éléments permanents de la population des follicules pileux. 
2. Variation du rapport $\frac{\mathrm{PL}+\mathrm{S}}{\mathrm{PC}}$

C'est en été que le rapport $\frac{\mathrm{PL}+\mathrm{S}}{\mathrm{PC}}$ est le plus faible : $37,0 \pm 1,8$ et en automne qu’il est le plus élevé : $51,5 \pm 1,5$; la différence de 28,2 p. 100 est hautement significative (tabl. 4). En hiver, le rapport $\frac{P L+S}{P C}$ ne diminue que de 2 unités, soit 3,8 p. 100 , vis-à-vis de celui de l'automne, la différence n'étant pas significative. Au printemps, la diminution du rapport $\frac{\mathrm{PL}+\mathrm{S}}{\mathrm{PC}}$ s'accentue, l'écart par rapport à l'automne atteignant 6,4 , soit 12,4 p. 100 , ce qui devient significatif. Mais la baisse de 4,4 du printemps par rapport à l'hiver précédent ne l'est plus.

\section{Les bourgeons pileux}

L'examen des coupes histologiques révèle la présence de bourgeons pilcux (b, fig. 1) dans les faisceaux de follicules pileux secondaires S. Ces bourgeons sont constitués par des amas de quelques cellules mises en évidence par la coloration rouge vif de leurs noyaux par le Rouge solide. Bien qu'ils soient inclus dans les faisceaux formés par les follicules secondaires, ces bourgeons pileux ne sont pas liés directement à une formation signifiant une production de poils : bulbes pileux ou poil lui-même. En outre, ils se situent à des niveaux différents dans le faisceau des follicules pileux, si bien qu'on ne peut les dénombrer exactement : toutefois, on les observe plus facilement en été, en raison de leur abondance, tandis qu'on les décèle difficilement aux autres saisons et même rarement en automne.

Par ailleurs, cet examen histologique des faisceaux de follicules pileux révèle que les PL producteurs de barbes, aisément reconnaissables au stade anagène, sont constamment présents. Il en résulte que les follicules pileux qui disparaissent avec leurs poils en été ne sont que des $\mathrm{S}$ producteurs de duvets.

\section{Discussion}

Les observations effectuées au cours d'un cycle annuel de production de poil confirment celles des éleveurs selon lesquelles le lapin angora fournit nettement moins de poil en été, soit environ 30 p. 100 de moins.

Comme nos lapins ont été soumis à un régime alimentaire constant, seuls les facteurs saisonniers peuvent être mis en cause dans cette variation de la production poilière : en l'occurrence, il s'agit du photopériodisme qui était contrôlé. Quant à la température, on sait qu'elle n'a pas d'action (Rougeot, 1957; 1961), sinon un effet modulatoire dans des cas exceptionnels (RoTHSCHILD, 1944 ; FLUX, 1970). Or, par précaution, nous avons évité qu'elle ne tombe en dessous de $10^{\circ} \mathrm{C}$. 
Tous les paramètres de la production pondérale, longueur et diamètre des poils, nombre de poils du pelage, ont varié : nous discutons point par point la signification de ces variations saisonnières.

\section{A. Poids des toisons}

La récolte des poils ayant poussé en été est donc nettement inférieure à celle d'automne et d'hiver : chez les 22 lapines observées, le poids de poils est en effet inférieur, en été, respectivement de 25 et 27 p. 100 par rapport à l'automne et à l'hiver; il ne représente que le $1 / 5$ (20,5 p. 100) de la récolte annuelle. Au printemps, le poids de poil se maintient au niveau moyen, avec juste le $1 / 4(25,5 \mathrm{p}$. 100) de la production annuelle que dépassent significativement les récoltes d'automne (26,8 p. 100) et d'hiver $(27,6$ p. 100).

Ces mesures pondérales, effectuées sur des périodes de croissance de poils identiques de 98 jours pour chaque saison, confirment non seulement les observations des éleveurs, mais celles consignées dans la littérature : ainsi, Kettner (1962) trouve des écarts moins prononcés que les nôtres entre les récoltes saisonnières, mais remarque que la tonte d'automne est la plus élevée avec 26,4 p. 100 de la production annuelle, tandis que celle d'été est la plus faible avec 24,2 p. 100 ; les pourcentages qu'elle donne pour les autres saisons sont respectivement de 25,1 pour la récolte d'hiver et de 24,3 pour celle du printemps. On peut expliquer cette atténuation des différences d'une part par une plus grande hétérogénéité des toisons (mâles et femelles confondus, provenant de 3 troupeaux différents; intervalles de récoltes moins rigoureux), d'autre part en raison du mode de récolte différent utilisé, la tonte. En effet, la tonte, non seulement modifie la croissance du poil et atténue le synchronisme du cycle folliculaire, mais intervient dans la sélection avec une tendance vers la prolongation de la durée de l'anagène et la réduction du télogène. En outre on sait que la composition et la structure du pelage des lapins angora allemands diffère de celle de la souche française avec un rapport

$\frac{\mathrm{PL}+\mathrm{S}}{\mathrm{PC}}$ plus élevé (environ 70, Koetter, 1967) et des jarres plus fins.

Le travail très exhaustif de KETTNER (1962) confirme lui-même des observations déjà anciennes (tabl. 5) : PiCKard (1930) calcule que les pourcentages des productions mensuelles dépassent 9 p. 100 de la production annuelle de septembre à janvier, tandis qu'ils sont en dessous de 7,5 p. 100 d'avril à juillet. FröLICH \& TegtmeYER (1939) obtiennent 17,9 p. 100 de la production annuelle à la tonte de juin, contre 36,2 p. 100 à celle de décembre. Plitt-Hardy \& Dolnick (1948) avec des lapins angora de très faible production obtiennent une production moyenne de $48,9 \mathrm{~g}$ en été contre 70,4 et $65,0 \mathrm{~g}$ respectivement en automne et en hiver, celle du printemps s'intercalant avec $58,1 \mathrm{~g}$. Mais en 1955, НABERBosch ne trouve plus de différences aussi marquées entre le semestre d'été et le semestre d'hiver pour les femelles $(256,2 \mathrm{~g}$ contre 263,2$)$ tandis qu'elle reste importante pour les mâles $(202,1 \mathrm{~g}$ contre 235,2$)$. Il est certes difficile de comparer des données prises selon des méthodes différentes sur des animaux très dissemblables, mais la tendance est malgré tout la même dans chaque cas avec une dépression de la production d'été qui semble aller en s'atténuant à mesure que les productions s'élèvent. Dans tous les cas (sauf pour Plitt-Hardy \& Dolnick, 1948), les récoltes ont été effectuées par tonte, ce qui — théoriquement - ne doit pas interférer avec l'induction des mues par les facteurs saisonniers. 


\section{TABleaU 5}

Poids de poils produits selon les saisons, chez diverses espèces.

Weight of hair produced according to season in some species.

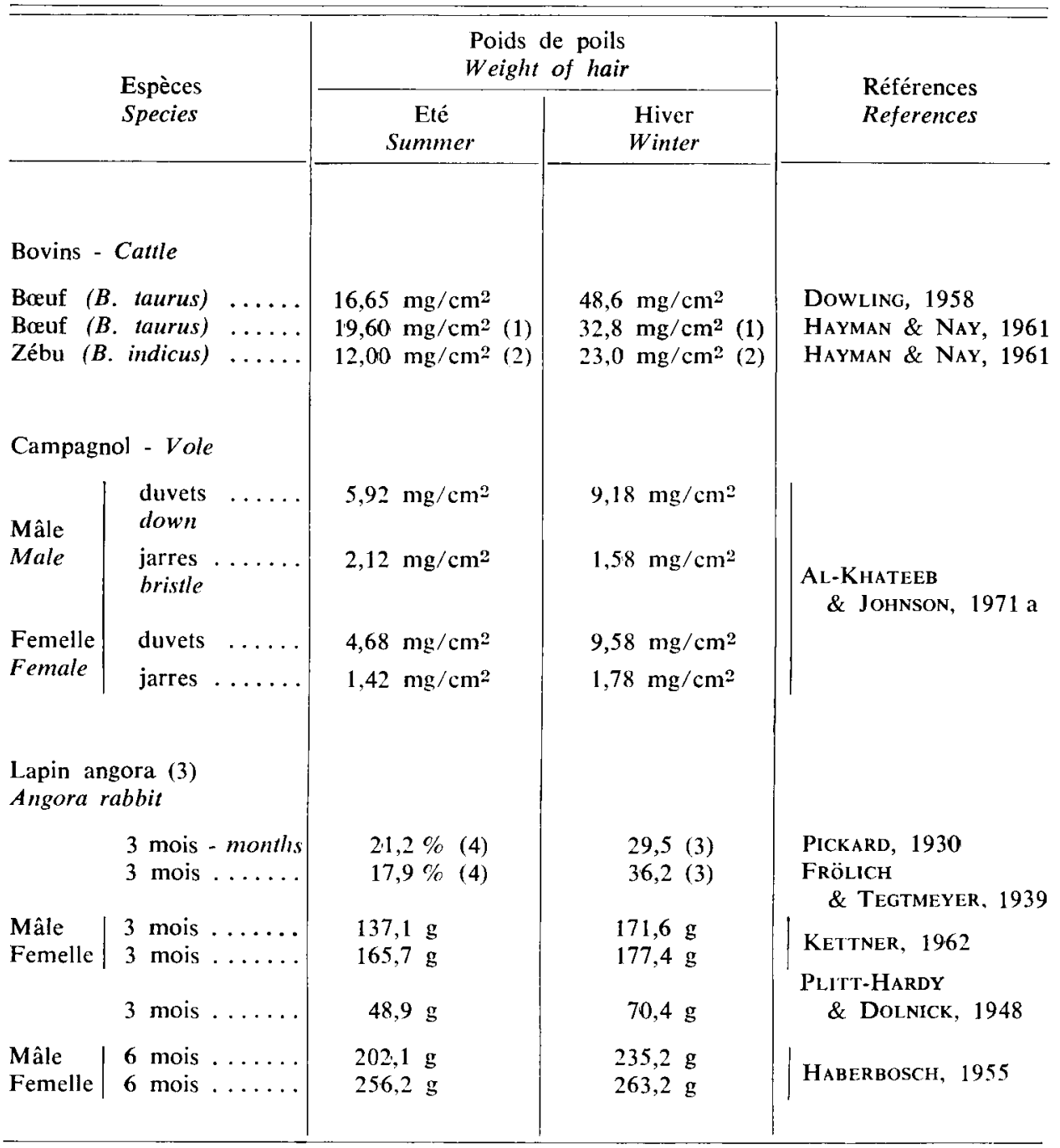

(1) Moyenne de 3 races - Mean of 3 breeds.

(2) Moyenne de 2 races - Mean of 2 breeds.

(3) Toison récoltée après une durée donnée de croissance de 3 ou 6 mois - Fleece sheared after a growth period of 3 or 6 months.

(4) p. 100 de la production annuelle - \% of yearly production. 


\section{Tableau 6}

Rapports des longueurs des poils du pelage d'hiver à celles du pelage d'été chez diverses espèces (calculs d'après données).

Ratio of winter fleece hair lenght to summer fleece hair length in some species.

\begin{tabular}{|c|c|c|c|}
\hline $\begin{array}{l}\text { Espèces } \\
\text { Species }\end{array}$ & $\begin{array}{l}\text { Poils de garde } \\
\text { Guard hair }\end{array}$ & $\begin{array}{l}\text { Sous poil } \\
\text { Underfur }\end{array}$ & $\begin{array}{l}\text { Références } \\
\text { References }\end{array}$ \\
\hline Cheval - Horse ...... & 2,10 & & Duerden \& Whitnale, 1930 \\
\hline Ane - Donkey ........ & 1,77 & & Duerden \& Whitnall, 1930 \\
\hline $\begin{array}{l}\text { Bouf (B. taurus) ..... } \\
\text { Cattle }\end{array}$ & 1,51 & & Duerden \& Whitnall, 1930 \\
\hline $\begin{array}{l}\text { Bœuf (B. taurus) .... } \\
\text { Cattle }\end{array}$ & 1,50 à $1,91(1)$ & & HAYMAN \& NAY, 1961 \\
\hline $\begin{array}{l}\text { Zébu (B. indicus) } \ldots . \\
\text { Zebu }\end{array}$ & 1,71 à $2,17(2)$ & & HAYMan \& NAY, 1961 \\
\hline Cerf - Red deer ...... & 1,40 & (3) & RYDER \& KAY, 1973 \\
\hline Cerf - Red deer ..... & 1,20 & (3) & RYDER, 1977 \\
\hline Chevreuil - Roe deer .. & 1,32 & & JOHNSON \& HORNBY, 1975 \\
\hline $\begin{array}{l}\text { Renard polaire } . . . . \\
\text { Artic fox }\end{array}$ & 3,02 & & UNDERWOOD \& REYNOLDS, 1980 \\
\hline Chat - Cat ........ & 1,20 & 1,25 & RYDER, 1976 \\
\hline Lièvre - Hare .... & 1,27 & 1,47 & HEwSON, 1963 \\
\hline Lièvre - Hare ... & 1,18 & 1,20 & BOROWSKI, 1964 \\
\hline Campagnol - Vole .... & 1,00 & & Al-Khateeb \& Johnson, 1971 \\
\hline $\begin{array}{l}\text { Musaraigne } \ldots \ldots \ldots \\
\text { Shrew mouse } \ldots \ldots\end{array}$ & 1,61 & & BOROWSKr, 1968 \\
\hline $\begin{array}{l}\text { Lapin angora } \ldots \ldots \\
\text { Angora rabbit }\end{array}$ & & 1,05 & KETTNER, 1962 \\
\hline $\begin{array}{l}\text { Lapin angora } \ldots \ldots \ldots \\
\text { Angora rabbit }\end{array}$ & 1,11 & 1.20 & Rougeot \& Thebault, p. 294 \\
\hline
\end{tabular}

(1) Moyenne de 3 races - Mean of 3 breeds.

(2) Moyenne de 2 races - Mean of 2 breeds.

(3) Absence de duvets en été - No down in summer. 
On possède peu de renseignements sur la variation saisonnière de la production pondérale de poils chez les autres espèces : elle double ou même triple chez les bovins, elle double chez le campagnol (tabl. 5). Les écarts entre pelage d'été et d'hiver peuvent donc être considérables et notamment plus élevés que chez le lapin angora, sans que ces deux seuls exemples permettent toutefois de généraliser.

Comment se comportent donc les divers paramètres de la production pondérale, longueur et diamètre des poils, nombre de follicules pileux en activité, dans ces variations saisonnières?

\section{B. Variation saisonnière des dimensions des poils}

\section{Longueurs}

Les longueurs des poils récoltés par épilation tous les 98 jours, lorsque le pelage atteint sa maturité, diffèrent selon les saisons. Elles sont significativement moindres en été par rapport à chacune des trois autres saisons dans chaque catégorie de poils, jarres, barbes et duvets. Par contre, les longueurs intermédiaires d'automne et de printemps ont tendance à se rapprocher des longueurs maximales qui se situent en hiver : mais si les différences ne sont pas significatives entre les longueurs intermédiaires et celles d'hiver pour les jarres, elles le sont quand même pour les barbes et les duvets. KettNer (1962) observe la même tendance chez ses lapins angora de souche allemande, dont le poil est récolté par tonte; la mesure portant sur 100 poils par mèche, jarres exclus; chez les lapines $(650 \mathrm{~g} / \mathrm{an}$ de poil), la moyenne des longueurs des poils du dos est minimale en été $(5,96 \mathrm{~cm})$ et maximale en hiver $(6,27 \mathrm{~cm})$, ce qui donne un rapport de longueurs hiver/été de 1,05, inférieur au nôtre; la différence peut provenir aussi bien de la souche que des modes de récolte ou de mesure. La longueur moyenne du printemps est intermédiaire $(6,16 \mathrm{~cm})$, tandis que celle de l'automne est plutôt voisine de celle d'été $(6,05 \mathrm{~cm})$.

Comme chez les autres espèces (tabl. 6), les poils d'hiver sont donc plus longs que ceux d'été, mais la différence semble moindre chez le lapin angora, les rapports étant de 1,11 pour les jarres et de 1,20 pour les duvets (1,05 d'après KeTtNER, 1962), alors qu'ils dépassent souvent 2 chez les ruminants et les solipèdes et atteint 3 chez le renard polaire (tabl. 6). Cette fonction adaptative de la longueur des poils aux changements saisonniers de la température se trouve accentuée chez les duvets qui ont pour rôle essentiel l'isolation thermique : ainsi l'écart des longueurs entre hiver et été ne dépasse pas 10 p. 100 pour les jarres de nos lapins angora; il atteint 15,2 p. 100 pour les barbes et 16,6 pour les duvets. Il en résulte une modification de la structure sur la longueur de la mèche où le duvet monte plus haut en hiver $(73,5$ p. 100) qu'en été $(67,5$ p. 100). Les quelques cas étudiés parmi les autres espèces (tabl. 6) montrent que cette modification adaptative doit être générale.

\section{Diamètres}

Les diamètres diminuent significativement en été dans les trois catégories de poils par rapport à chacune des trois autres saisons : c'est la seule variation nette observée. Aux trois autres saisons, les diamètres se rapprochent de la valeur la plus élevée qui est atteinte au printemps pour les jarres et les barbes, en hiver pour les duvets; mais les différences entre les diamètres des poils ayant poussé durant ces trois saisons ne sont pas toujours significatives. Comme pour les longueurs, on remarque que l'écart s'accroît entre le diamètre saisonnier le plus élevé et le diamètre minimal do l'été à 
mesure que l'on passe de la catégorie de poil la plus grossière à la catégorie la plus fine : 3 p. 100 pour les jarres, 5 p. 100 pour les barbes, 6 p. 100 pour les duvets.

Cette variation saisonnière du diamètre modifie la compacité du pelage ct ajuste ainsi son pouvoir isolant aux changements saisonniers de la température, d'autant plus qu'il faut plutôt tenir compte de la section droite des poils que de leur diamètre dans cet effet de volume : en faisant l'approximation de considérer la section droite des poils comme circulaire, les écarts précédents deviennent respectivement 6,5 p. 100 pour les jarres, 10,3 p. 100 pour les barbes et 11,3 p. 100 pour les duvets. JoHnson \& HoRnby (1975) démontrent l'importance de l'effet isolant dû à l'augmentation du diamètre des jarres dans le pelage d'hiver du chevreuil : il est vrai que dans ce cas l'augmentation du diamètre est particulièrement élevée, puisque celui-ci double par rapport à l'été $(205 \mathrm{fm}$ contre $107 \mathrm{fm})$. Ces auteurs remarquent en outre que l'augmentation du diamètre s'effectue uniquement par l'intermédiaire de la moelle, si bien que les jarres d'hiver ne pèsent pas plus que ceux de l'été, processus qui économise la kératine et évite le port d'un pelage de poids excessif. Il apparaît par contre que le duvet intervient de façon mineure dans la régulation saisonnière de l'isolation thermique chez les cervidés : il n'existe qu'en hiver où il reste très épars dans le pclage (RYDER \& KAY, 1973 ; JOHNSON \& HORNBY, 1975).

Chez le lapin angora les trois catégories de poils sont médullées; mais nous ne possédons aucune méthode suffisamment rigoureuse pour savoir dans quelle proportion le volume de la moelle peut varier saisonnièrement.

Signalons simplement qu'en règle générale, pour un type de poil donné, le diamètre de la moelle varie dans le même sens que celui du poil, les dimensions de ces deux diamètres dépendant de la taille de la papille dermique (Rudall, 1955; Trigg, 1972). Nos observations sur la croissance du poil du lapin angora (Thebault \& Rougeot, données non publiées) vont dans ce sens : pendant la $13^{\mathrm{e}}$ semaine après l'épilation, la croissance pondérale des poils diminue de 40 p. 100 par rapport au maximum atteint à la $4^{\circ}$ semaine, tandis que la croissance en longueur ralentit de 23 p. 100 et que la section droite se rétrécit de 50 p. 100 . Malgré les erreurs qui affectent l'estimation de la mesure (corrections pour les irrégularités de contour, notamment les angles rentrants; fig. 1), la disproportion demeure. On peut donc penser que la moelle participe ainsi pour une part importante dans la variation saisonnière du diamètre du poil angora.

En outre, comme nous allons le constater (tabl. 7), la diminution du diamètre du poil en été et son augmentation en hiver ne semblent pas générales, le mécanisme de régulation du pouvoir isolant par la variation du diamètre paraissant dépendre de la structure du pelage et des conditions du milieu, même chez le lapin angora.

Ainsi, Haberbosch (1955) trouve que les poils d'été du lapin angora sont un peu plus grossiers que ceux d'hiver (tabl. 7), de façon significative pour les mâles dont la production de poils est de $440 \mathrm{~g} / \mathrm{an}$ et non significative pour les femelles dont la production s'élève à $520 \mathrm{~g} / \mathrm{an}$ ). De même, KeTTNER (1962) relève des différences «extrêmement faibles» entre les diamètres d'été et ceux d’hiver qui ont tendance à leur être inférieurs (tabl. 7). Cependant, KetTnER signale des différences selon les troupeaux. Ainsi les lapins de Halle fournissent les poils les plus grossiers au printemps et les plus fins en automne. Ces résultats sont de toute façon en contradiction avec nos observations. Or, on ne peut expliquer ces désaccords par des différences de souche, notamment la plus grande finesse des duvets, ni par le mode de récolte de la toison, la tonte. Par contre, il semble que les méthodes de mesure et d'élevage utilisés par 
les auteurs allemands puissent être la cause de cette inversion des diamètres saisonniers. Ainsi, ils ne précisent pas s'ils effectuent leurs mesures en atmosphère contrôlée, surtout au point de vue humidité de l'atmosphère (Anderson \& Palmer, 1948), ou bien dans les laboratoires sans conditionnement d'air. Or l'humidité relative de l'atmosphère passe facilement de 40 p. 100 en hiver, lorsque le local est chauffé, à plus de 70 p. 100 en été, ce qui a pour effet d'augmenter de plus de 4 p. 100 le diamètre des poils (ROugEot, 1953). Au point de vue élevage, les lapins allemands reçoivent une alimentation mixte comprenant des granulés et 300 à $500 \mathrm{~g} / \mathrm{j} /$ lapin d'aliments aqueux, betteraves ou verdure; or, la qualité et l'efficacité de ces aliments varient selon les saisons et l'on sait que le diamètre des poils longs est très sensible aux variations du régime alimentaire (laine du mouton : DownEs \& SHARry, 1971). Enfin, aucune mention n'est fait sur le rythme lumineux des locaux d'élevage.

\section{TABleaU 7}

Modifications du diamètre des poils en fonction des saisons chez diverses espèces (en $\mu \mathrm{m}$ ). Seasonal changes in hair diameter in some species (in $\mu \mathrm{m}$ ).

\begin{tabular}{|c|c|c|c|}
\hline $\begin{array}{l}\text { Espèces } \\
\text { Species }\end{array}$ & $\begin{array}{c}\text { Eté } \\
\text { Summer }\end{array}$ & $\begin{array}{l}\text { Hiver } \\
\text { Winter }\end{array}$ & $\begin{array}{l}\text { Références } \\
\text { References }\end{array}$ \\
\hline Cheval - Horse .......... & 68,90 & 60,24 & Duerden \& Whitnalle, 1930 \\
\hline Ane - Donkey ........... & 78,23 & 77,82 & Duerden \& Whitnall, 1930 \\
\hline Bœuf (B. taurus) & 51,00 & 50,88 & Duerden \& Whitnall, 1930 \\
\hline Bœuf (B. taurus) & 38,50 & 43,60 & Dowling, 1958 \\
\hline Bœuf (B. taurus) & $64,30(1)$ & $63,00(1)$ & Hayman \& NaY, 1961 \\
\hline Zébu (B. indicus) - Zebu .... & $73,00(2)$ & 61,00 & Hayman \& NaY, 1961 \\
\hline Chevreuil - Roe deer ...... & 107,00 & $2,05,00$ & JOHNSON \& HORNBY, 1975 \\
\hline $\begin{array}{l}\text { Campagnol - Vole } \\
\text { mâle }- \text { male } \ldots \ldots \ldots \ldots \\
\text { femelle - female } \ldots \ldots \ldots\end{array}$ & $\begin{array}{l}15,29 \\
13,20\end{array}$ & $\begin{array}{l}10,60 \\
10,10\end{array}$ & AL-KhateEb \& JohnSON, 1971 a \\
\hline $\begin{array}{l}\text { Lapir angora (duvets) } \\
\text { Angora rabbit (down) } \\
\text { mâle }- \text { male } \ldots \ldots \ldots \ldots \\
\text { femelle }- \text { female } \ldots \ldots \ldots\end{array}$ & $\begin{array}{l}12,90 \\
12,70\end{array}$ & $\begin{array}{l}11,90 \\
12,30\end{array}$ & HaBERBOSCH, 1955 \\
\hline $\begin{array}{l}\text { mâle }- \text { male } \ldots \ldots \ldots \ldots \\
\text { femelle }- \text { female } \ldots \ldots\end{array}$ & $\begin{array}{l}12,08 \\
12,48\end{array}$ & $\begin{array}{l}11,53 \\
11,71\end{array}$ & KETTNER, 1962 \\
\hline $\begin{array}{l}\text { Lapin angora (femelle) } \\
\text { Angora rabbit (female) } \\
\text { jarres - bristle } \ldots \ldots \ldots \ldots \\
\text { barbes }- \text { awn } \ldots \ldots \ldots \ldots \ldots \\
\text { duvets }- \text { down } \ldots \ldots \ldots \ldots \ldots\end{array}$ & $\begin{array}{l}58,06 \\
29,18 \\
16,06\end{array}$ & $\begin{array}{l}58,92 \\
30,24 \\
17,06\end{array}$ & Rougeot \& Thebault (tabl. 3) \\
\hline
\end{tabular}

(1) Moyenne de 5 races - Mean of 5 breeds.

(2) Moyenne de 2 races - Mean of 2 breeds. 
Si l'on se réfère aux différences de diamètres des poils entre pelage d'hiver et pelage d'été chez les autres espèces (tabl. 7), on retrouve des contradictions semblables qui relèvent aussi bien du mode d'adaptation à un milieu donné de la composition et de la structure du pelage que des méthodes d'observations. Nous avons mentionné que le pouvoir isolant du pelage d'hiver des cervidés était accru grâce au doublement du diamètre des jarres par rapport au pelage d'été. Il semble qu'il en soit de même chez le bœuf des zones tempérées fraîches (Duerden \& Whitnall, 1930 ; Dowling, 1958). Par contre, dans les zones chaudes ou tempérées chaudes, la tendance est inversée avec une augmentation très atténuée du diamètre chez le bœuf (Bos taurus) introduit dans ces pays, alors que la différence est très accentuée chez le zébu (Bos indicus) ou autres races tropicales (Dowling, 1958, 1959 ; Berman \& Volcani, 1961 ; Hayman \& NaY, 1961 ; Hayman, 1965). Or il faut remarquer que les pelages sont peu denses en zone tropicale, particulièrement chez les espèces indigènes : en Egypte, le nombre de poils est de 394/ $\mathrm{cm}^{2}$ chez le buffle et de 2633 chez le bœuf, d'après HafEz, BADRELDIN \& SHAFEI (1955). Les poils courts, grossiers, fortement médullés et épars du pelage d'été permettent une ventilation efficace de la peau chez les bovins vivant en pays chaud, tout en constituant un écran contre le rayonnement solaire. Par contre, en zone tempérée fraîche, l'adaptation du pelage du bœuf aux rigueurs de l'hiver par immobilisation d'une importante masse d'air entre les poils s'opère plus par l'allongement des poils et l'augmentation du nombre des duvets que par l'augmentation du diamètre qui est minime (Yeates, 1955; Dowling, 1958, 1959 ; Johnson, 1977 b).

Duerden \& Whitnall (1930) trouvent aussi que les diamètres augmentent en été chez le cheval et chez l'âne sous climat tempéré en Afrique du Sud. Mais ils calculent la moyenne de l'ensemble des poils, jarres et duvets confondus et comme l'on sait que les duvets sont plus nombreux en hiver, le diamètre moyen paraît surestimé. Il se pourrait que la même erreur puisse être imputée aux mesures de HABERBosch (1955) et de Ketrner (1962) chez le lapin angora dans la mesure où il est difficile d'affirmer qu'on élimine toutes les barbes de la mèche chez les animaux tondus.

Il serait tentant de comparer plutôt le pelage du lapin angora à la toison du mouton dont la croissance est de longue durée, sinon permanente. Si l'on s'adresse, en effet, à des toisons de type primitif, le diamètre de la laine et des autres poils varie saisonnièrement avec un affinement en hiver (Doney \& SMITH, 1961, chez le blackface ; Priestley, 1967, chez le herdwick) : mais, en ce cas, on ne départage pas l'action possible de facteurs du milieu variant saisonnièrement, comme le photopériodisme, de l'action de la tonte toujours effectuée au printemps et qui modifie l'activité folliculaire. De fait, Priestley (1967) observe que la réduction du diamètre des poils de Herdwick en hiver est équilibrée par l'augmentation du volume de la gaine interne fournie également par le bulbe pileux. Donc, comme pour la moelle des jarres chez le chevreuil, on se trouve en présence d'un mécanisme de régulation qui modifie le diamètre du poil en répartissant simplement de façon différente les cellules en provenance du bulbe pileux et en modifiant leur taille. Est-ce un système analogue qui intervient chez le Campagnol où l'épaississement des jarres en été et leur affinement en hiver serait sous contrôle endocrinien (AL-Khateeb \& Johnson, 1971 a), sans qu'on puisse toutefois exclure complètement l'action du facteur nutritionnel ? De toute façon, étant donné l'importance de la moelle dans la variation du diamètre du poil, l'influence de ce paramètre, qui d'ailleurs ne varie qu'entre d'étroites limites chez le lapin angora, ne doit pas jouer beaucoup dans la variation saisonnière du poids de la toison. Comme la variation de la longueur paraît en outre relativement modérée (10 à 16 p. 100), celle du volume des poils n'apparaît pas comme le facteur principal de la variation pondérale 
saisonnière du pelage du lapin angora. Il reste donc à examiner la variation saisonnière du nombre des duvets.

C. La variation saisonnière du nombre de duvets : le creux estival Le rapport $\frac{\mathrm{PL}+\mathrm{S}}{\mathrm{PC}}$ subit des variations importantes en fonction des saisons.

Il atteint le maximum de sa valeur, de lordre de 50 pour nos lapins angora, en automne et en hiver. Puis il amorce une baisse de 12 p. 100 au printemps et enfin tombe au plus bas en été, à 37 ; la chute est donc d'environ 30 p. 100 par rapport à l'automne. Cette chute estivale traduit la suppression de plus de $1 / 4$ des follicules pileux secondaires ainsi que des duvets qu'ils produisent. Cette régression de la population des duvets en été est donc le principal facteur de la diminution de poids que subit la toison en cette saison et d'autant plus qu'il ne semble pas que ce soit sur le dos que le manque de duvets en été soit le plus important : l'observation du pelage montre en effet que la cuisse, notamment, subit une perte de poils plus prononcée.

\section{Tableau 8}

Modification du nombre de poils par unité de surface, ou par groupe folliculaire en fonction de la saison, chez diverses espèces.

Changes in the number of hairs per surface unit of per follicle group according to season in various species

\begin{tabular}{|c|c|c|c|}
\hline $\begin{array}{l}\text { Espèces } \\
\text { Species }\end{array}$ & $\begin{array}{c}\text { Eté } \\
\text { Summer }\end{array}$ & $\begin{array}{l}\text { Hiver } \\
\text { Winter }\end{array}$ & $\begin{array}{l}\text { Références } \\
\text { References }\end{array}$ \\
\hline \multicolumn{3}{|l|}{ Campagnol - Vole } & \multirow{3}{*}{ Al-KhATEEB \& JOHNSON, 1971 a } \\
\hline \begin{tabular}{l|l} 
Poil - Hair/ & mâle ..... \\
$1,54 \mathrm{~mm}^{2}$ & femelle..
\end{tabular} & $\begin{array}{l}153 \\
147\end{array}$ & $\begin{array}{l}245 \\
272\end{array}$ & \\
\hline \begin{tabular}{l|l} 
Poils/gr. foll. & mâle .... \\
Hair/foll. gr. & femelle ..
\end{tabular} & $\begin{array}{l}5,97 \\
6,73\end{array}$ & $\begin{array}{l}9,47 \\
8.61\end{array}$ & \\
\hline Lièvre - Hare & & & \\
\hline $\begin{array}{l}\text { Poil/faisceau - Hair/bundle . } \\
\text { Poils/cm² - Hair } / \mathrm{cm}^{2} \ldots \ldots\end{array}$ & $\begin{array}{c}40-50 \\
6880-8600\end{array}$ & $\begin{array}{c}40-90 \\
6720-15 \quad 120\end{array}$ & BOROWSKI, 1964 \\
\hline Chevreuil - Roe deer ...... & $\begin{array}{l}\text { pas de du } \\
\text { no down }\end{array}$ & $\begin{array}{l}\text { vet en été } \\
n \text { summer }\end{array}$ & JOHNSON \& HORNBY, 1975 \\
\hline$\frac{\mathrm{PL}+\mathrm{S}}{\mathrm{PC}}$ & 37,0 & 49,5 & Rougeor \& Thebault, (tabl. 4) \\
\hline
\end{tabular}


Cette suppression d’une proportion élevée de duvets en été, appelle plusicurs remarques.

Chez les autres espèces (tabl. 8), les observations demeurent rares. Chez le lièvre (Borowski, 1964), le nombre total de follicules pileux, comptés dans les groupes folliculaires du dos, tombe de 40-90 en janvier à 40-50 en juillet; chez le campagnol (Al-Khateeb \& Johnson, 1971 a), le nombre de follicules pileux secondaires producteurs de duvets, comptés aussi par groupe folliculaire sur le dos, baisse de 9,47 en hiver à 5,97 (-37 p. 100) en été chez le mâle et de 8,61 à 6,73 chez la femelle ( -22 p. 100). Chez le vison, le nombre de duvets présents dans le pelage en été est inférieur de 32 p. 100 à celui de l'hiver (Allain \& Rougeot, 1980 ; Allain, Martinet \& Rougeor, 1981 ; Rougeot, Thebault \& Allain, 1982). Chez les cervidés (cerf : RYDER \& KAY, 1973 ; chevreuil : JohNSON \& HoRNBY, 1975), le comptage devient inutile, le rare duvet d'hiver ayant à peu près ou totalement disparu en été.

Ainsi la réduction du nombre de duvets dans le pelage d'été peut atteindre des proportions élevées, confirmant l'observation courante de la chute spectaculaire des duvets à la mue de printemps par opposition à celle, à peine perceptible, à la mue d'automne. Cette différence du nombre de duvets contenus dans les pelages d'été et d'hiver apparaît même comme le critère principal pour différencier ces deux pelages (Allain \& Rougeot, 1980 ; Rougiot, Thebault, Allain, 1982).

Or si l'on considère l'importance de ce phénomène dans l'adaptation du pelage aux modifications saisonnières des facteurs climatiques, on demeure étonné, aussi bien par la rareté des données que par labsence dexplication du processus. Il est vrai que la notion du cycle folliculaire selon laquelle les poils sont remplacés un par un dans un follicule pileux permanent (DRY, 1926 ; CHASE, 1954) ainsi que la théorie de labsence de néogénèse folliculaire chez ladulte (Muller, 1971) n'offrent guère de possibilité d'explication.

\section{Le processus de la modification saisonnière du nombre de follicules pileux produisunt des duvets}

En fait, on peut expliquer au moins en partie la maigreur du pelage d'été, par le déroulement étalé dans le temps des cycles folliculaires. En effet, dans ce système, l'ancien poil tombe par digestion de sa racine lorsque la pointe du nouveau poil atteint le canal pileux ou fait juste éruption à la surface de la peau (anagène $V$ ) : il en résulte qu'il manque effectivement des poils dans le pelage à ce moment et la structure du pelage ne sera achevée que lorsque tous les poils auront atteint leur taille définitive, leurs follicules pileux étant en télogène. On dit que le pelage est mûr. Dans la mue de printemps, les cycles folliculaires se succèdent de façon assez étalée, si bien que le pelage qui comporte des poils de toutes les tailles, paraît maigre par rapport à celui de l'hiver, outre que la taille finale des poils est plus courte en hiver. C'est d'ailleurs ainsi que se constitue le pelage d'été des espèces à mue annuelle (ovins : Rougeot, 1961 ; bovins, Berman \& VolCani, 1961).

Chez les espèces à deux mues annuelles - au printemps et à l'automne - le pelage stable d'été n'existe que pendant quelques semaines ( 3 semaines chez le vison; Bassett \& Llewellyn, 1949 ; Allain \& Rougeot, 1980). Mais le remplacement du poil par le processus du cycle folliculaire ne peut rendre compte de l'absence d'une proportion élevée de duvets en été et de la brutalité du changement. Si Bonowski (1964) 
et surtout Al-Kuateeb \& Johnson (1971 a) ont bien décrit la disparition et la reconstitution des follicules pileux, ils l'ont fait sans autre commentaire.

En fait, nous nous sommes aperçus que les follicules pileux qui apparaissent et disparaissent saisonnièrement appartiennent à une catégorie particulière, les follicules pileux dérivés : ils sont ainsi nommés (HARDY \& LYNE, 1956) parce qưils se forment par bourgeonnement sur d'autres follicules pileux qui se sont constitués, cux, à partir de l'épiderme primitif du fœetus. Selon nos analyses de travaux antérieurs (Dromidova, 1954 ; HARDY \& I.YNF, 1956; LYNE, 1957, 1966) et nos propres observations sur le mouton (Rougeot, 1974) et le lapin angora (THEBAult, 1977), ces follicules pileux épidermiques qui comprennent tous les primaires et au moins les premiers secondaires formés, cessent de se multiplier dès les premiers signes de kératinisation dans l'épiderme primitif. En particulier, aucun canal pileux, qui permet le passage du poil à travers l'épiderme définitif n'est créé par la suite : aussi, les poils des follicules pileux dérivés traversent-ils l'épiderme par le canal pileux commun construit par le follicule épidermique qui les produit. Il en résulte que le follicule pileux ćpidermique constitue avec ses follicules dérivés une véritable unité folliculaire, le follicule pileux composé (Rougeot, 1981 ; Rougeot, Thebault \& Allain, 1982). Ce follicule pileux est plus qu'une simple curiosité anatomique fournissant plus de laine (mouton : Hardy \& LYNE, 1956) ou de duvets (lapin : Whiteley, 1958) mais remplit des fonctions précises (Rougrot, 1981 ; Rougeot, Thebault \& Allain, 1982) : a) la multiplication des follicules pileux dérivés permet d’assurer une couverture pileuse suffisante chez les espèces où la phase de formation des follicules épidermiques est brève $(15$ jours chez le lapin ; Kontter, 1965 ; Rusaouën, 1966 ; Thebaul.t, 1977 ; par contre, 30-40 jours chez le mouton : CARTER, 1943) et où l'augmentation du poids corporel, donc de la surface de la peau, est considérable par rapport à ceux de la naissance (poids multiplié par 80 à 100 chez le lapin, 100 à 200 chez le vison). Il faut rappcler que chez le lapin (lapin angora : ThEBAult, 1977), la quasi totalité des follicules dérivés se forment après la naissance; b) la variation du nombre de follicules pileux dérivés permet l'adaptation de la structure du pelage aux variations climatiques saisonnières (ALLAIN \& RouGeot, 1980 ; Rougeot, 1981 ; Rougeot, Thebault \& Allain, 1982). Dans le cas du lapin angora, où la longueur des poils amoindrit, sinon élimine, le rôle d'adaptation du pelage - cette longueur des poils est plutôt un inconvénient - la fonction demeure.

Peut-on considérer comme une néogénèse la reconstitution des follicules pileux dérivés lors de la mue d'automne? Il ne semble pas qu'on puisse admettre cette vue dans la mesure où ces follicules dérivés font partie intégrante du follicule pileux composé dont ils expriment l'activité générale au même titre que la croissance en longueur ou le diamètre du poil et que le remplacement cyclique du poil. Il semble d'ailleurs que la partie permanente du follicule pileux épidermique présente des propriétés particulières de prolifération : InABA, ANTONY \& MCKInSTRY (1979) constatent, entre autres, que la gaine externe au niveau des glandes sébacées, à condition que cette glande ne soit pas supprimée et que l'infundibulum reste intact, peut régénérer chez l'homme des follicules pileux fonctionnels. Les cas signalés de néogénèse des follicules pileux restent donc l'exception et demeurent discutés (STraile, 1959 ; LyNe \& Brook. 1964 ; Muller, 1971).

\section{La régulation saisonnière de l'activité folliculaire}

Comme nous l'avons mentionné dans l"introduction, l'épilation induit une reprise de l'activité folliculaire qui s'opère en strict synchronisme. Mais le fait remarquable 
et jamais encore signalé est qu'une proportion appréciable des follicules pileux dérivés, 12 p. 100 au printemps, 25 p. 100 en été, échappent totalement à cette action en maintenant leur involution saisonnière. Or, il était notoirement admis jusqu'à présent que l'épilation remettait en activité la totalié des follicules pileux : cette déduction provenait d'observations faites sur la souris et le rat de laboratoire qui sont des espèces non saisonnières et dont le pelage, très lâche, ne semble comporter aucun ou très peu de follicules pileux dérivés.

Nous avons, par ailleurs, montré que l'influence de la saison retentit aussi sur les follicules pileux qui fonctionnent selon un cycle et dont l'activité est induite par l'épilation puisqu'ils fournissent des poils dont les dimensions, longueur et diamètre, sont fonction de l'époque de l'année. Cependant, nous ne pouvons fournir d'explication satisfaisante à la présence de follicules primaires centraux en télogène, peu de temps après l'épilation et à l'augmentation significative de leur nombre en été : s'agissait-il de poils dont les follicules pileux étaient au repos au moment de l'épilation et qui se sont cassés lors de la récolte? ou bien de follicules pileux ayant une phase d'anagène extrêmement brève? On sait que la mutation angora porte sur l'allongement de la durée de l'anagène, mais de façon variable selon les follicules pileux, même à l'intérieur d'un même pelage.

Ainsi les variations saisonnières de la structure du pelage du lapin angora, qui apparaissent comme tout à fait normales lorsque la toison est récoltée par tonte (Frölich \& Tegtmeyer, 1939 ; KettNer, 1962 ; Haberbosch, 1955) sont maintenues même lorsque le poil est récolté par épilation. Cette opération n'a donc aucun effet sur l'action d'un photopériodisme qui, on le sait, est le facteur saisonnier de la régulation des mues du pelage (Bissonnette, 1935 ; Bissonnette \& Wilson, 1939 ; Belayev \& UtKin, 1949 ; Yeates, 1955 ; Rougeot, 1957, 1961 ; Harvey \& McFari.ane, 1958 ; Al-Khateeb \& Johnson, $1971 \mathrm{~b}$; Rougeot, Allain \& Martinet, 1982) et nous avons observé qu'il en était de même chez le lapin à pelage normal (Rougeot \&. Thebaulr, données non publiées).

L'intervention du relai endocrinien est encore mal définie. On connaît, certes, depuis longtemps l'action des hormones thyroïdiennes et des stéroïdes sexuels et surrénaliens sur les cycles folliculaires (MoHN, 1958 ; EBLING \& JoHNson, 1964) et sur la croissance de la laine (Ferguson, Wallace \& Linder, 1965), mais l'interprétation de leur action dans les changements saisonniers du pelage demeure difficile (JoHNson, 1977 a). Cependant, la mue d'automne est indubitablement induite par la mélatonine (hermine : Rust \& Meyer, 1969 ; vison : Allain \& Rougeot, 1980 ; Allain, MarTinet \& Rougeot, 1981; Rougeot, Thebault \& Allain, 1982) tandis que la prolactine induit la mue de printemps (vison : Allain, Martinet \& Rougeot, 1981) sans qu'on sache si ces deux hormones agissent directement ou par l'intermédiaire d'autres hormones. Pour notre part, nous avons montré que chez le lapin angora l'administration en été de mélatonine induit également un pelage d'automne-hiver (Rougeot \& Thebault, données non publiées).

\section{Conclusion}

La croissance de la toison du lapin angora subit des variations saisonnières réglées par le photopériodisme, comme tous les pelages. Ces modifications portent d'une part sur les dimensions des poils, longueurs et diamètres, accompagnées très proba- 
blement de changements dans les proportions de moelle, d'autre part sur le nombre de duvets produits par les follicules pileux secondaires dérivés. Il en résulte une toison longue et fournie en automne-hiver par contraste à une toison significativement plus courte et plus lâche en été.

Ainsi la toison du lapin angora conserve les caractères d'adaptation des pelages aux changements saisonniers des facteurs du climat, malgré l'allongement considérable de ses poils, lié au prolongement de l'anagène, qui lui ôte toute signification adaptative.

L'épilation, qui est pratiquée tous les 3 mois pour récolter le poil, exerce son effet d'induction de l'anagène bien connu, en relançant la réponse en synchronisme de la toison. Mais les changements saisonniers structuraux de la toison ne sont pas affectés pour autant. En particulier l'épilation n'a aucune action sur les fluctuations saisonnières de la population des follicules pileux secondaires dérivés dont les séquences de régressions, complètes, avec perte des poils, puis de reconstitutions, sont sans aucun rapport avec le cycle folliculaire classique.

Ces observations nous conduisent à réviser le schéma du processus des changements saisonniers de la composition et de la structure des pelages en faisant appel à la notion de follicule pileux composé, défini par la présence d'un seul canal pileux commun pour le passage à travers l'épiderme des poils fournis par les follicules pileux composants. Dans cette unité folliculaire, les variations saisonnières du nombre de follicules pileux dérivés, très importantes puisqu'elles portent sur un tiers d'entre eux dans les cas examinés, font partie intégrante de lactivité générale du follicule pileux au même titre que les variations saisonnières en longueur et en diamètre des poils. On ne peut donc attribuer, en particulier, une signification de néogénèse à la multiplication des follicules pileux dérivés à la mue d'automne.

Il résulte enfin de ces observations et pour revenir à la production de poils chez le lapin angora, qu'on ne peut remédier à la dépression estivale de la croissance de sa toison que par un traitement photopériodique ou hormonal.

Accepté pour publication en avril 1983.

\section{Summary}

\section{Seasonal variations in pelage composition and structure with special reference to Angora rabbit}

Seasonal variations in angora rabbit wool production - weight, length and diameter of the three types of hair and hair number per hair follicle group - were observed every 98 days at each plucking time (in summer, autumn, winter and spring) in 22 adult Angora does outside the reproductive period. The animals were kept on straw litter in individual hutches in a closed building where the minimum temperature was $10^{\circ} \mathrm{C}$ and the artificial lighting regulated according to the natural photoperiod by a photoelectric system. The diet, constant all year long, was given in the form of pellets, supplying 17 p. 100 crude proteins, 16 p. 100 crude fibre and $2700 \mathrm{kcal} / \mathrm{kg}$ of digestible energy.

Seasonal variations in coat composition and structure, regulated by photoperiodism, were observed despite the longer growth period of angora hair and the inducing effect of plucking on hair follicle activity. The weight of the angora wool obtained (tabl. 1) was minimal in summer $(20 \mathrm{p}$. 100 of the annual production, mean weight : $860 \mathrm{~g})$, maximal in autumn $(26.8 \mathrm{p} .100)$ and in winter $(27.6 \mathrm{p} .100)$ and intermediate in spring (25.5 p. 100). All weight components varied in the same way : 1) summer/winter differences in length (tabl. 2) were 9.6 p. 100 in guard hair, 15.9 p. 100 in awns and 16.6 p. 100 in 
downs, so that underfur was higher in the winter staples $(73.5$ p. 100) than in those of the summer $(67.5$ p. 100);2) summer/winter differences in diameter (tabl, 3) increased with the fineness of the hair classes : 3,5 and $6 \mathrm{p}$. 100 respectively; 3 ) the hair follicle population was maximal in autumn and winter (non-significant difference, tabl. 4). A part of the secondary follicles of downs (S) disappeared in spring and even more in summer, whereas lateral primary follicles of awns (PL), and especially central primary follicles of

guard hair (PC), used as markers, were permanent features (fig. 1); the ratio $\frac{\mathrm{PL}+\mathrm{S}}{\mathrm{PL}}$

(tabl. 4) decreased by 12.4 p. 100 in spring and by 28.2 p. 100 in summer. Histological observations showed that only derived secondary hair follicles, formed by budding from epidermal hair follicles, were affected by seasonal regression and renewal : this process is quite different from the classic hair follicle cycle and has no neogenetic significance.

These observations allowed to determine the compound hair follicle structure and two important functions of this follicular unit : underfur development in growing mammals and adaptation of the adult coat to seasonal climatic variations. Results were compared to other data on seasonal angora wool production and on seasonal coat changes in other species (tabl. 5, 6, 7, 8).

\section{Références bibliographiques}

Allain D., Rougeot J., 1980. Induction of autumn moult in mink (Mustela vison Peale and Beauvois) with melatonin. Reprod. Nutr. Dev., 20, 197-201.

Allain D., Martinet L., Rougeot J., 1981. Effect of melatonin implants on changes in the coat, plasma prolactin level and testis cycle in the mink (Mustela vison). In : Ortavant R., Pelletier J., Ravault J.P., Photoperiodism and reproduction in vertebrates, Colloques de l'I.N.R.A., 6, 263-271. Inst. Nat. Rech. Agron., Paris.

Anderson S.L., Palmer R.C., 1948. Effect of moisture on wool fineness measurement. I.W.T.O., Techn. Com. Proc., 2, 5-12.

Bassett C.F., Llewellyn L.M., 1949. The molting pattern and fur growth pattern in the adult mink. Am. Midl. Nat., 48, 751-757.

Bassett C.F., Pearson O.P., Wilke F., 1944. The effect of artificially-increased length of day on molt, growth and priming silver fox pelts. J. exp. Zool., 96, 77-83.

Belyaev D.K., UtKin L.G., 1949. Effet du raccourcissement de la durée du jour sur les délais de la maturité de la fourrure (en russe). Karakulevod. Zverodvod., 1949 (2), 59-62.

Berman A., Volcani R., 1961. Seasonal and regional variation in coats characteristics of dairy cattle. Aust. J. agric. Res., 12, 528-538.

Bissonnette T.H., 1935. Relations of hair cycles in ferrets to changes in the anterior hypophysis and light cycles. Anat. Rec., 63, 159-168.

Bissonnette T.H., Bailey E.E., 1944. Experimental modification and control of moults and changes of coat-color in weasels by controlled lighting. Ann. N.Y. Acad. Sci., 45, 221-260.

Bissonnette T.H., WiLson E., 1939. Shortening daylight periods between May 15 and September 12 and the pelt cycle of the mink. Science, 89, 418-419.

Borowski S., 1964. Studies on the european hare. I - Moulting and coloration. Acta theriol., 9, 217-231.

Borowski S., 1968. On the moult of the common shrew. Acta theriol., 13, 483-498.

Butcher E.O., 1934. The hair growth in the albino rat. Anat. Rec., 61, 5-19.

Butcher E.O., 1951. Development of the pilary system and the replacement of hair in mammals. Ann. N.Y. Acad. Sci., 53, 508-516. 
Carter H.B., 1943. Studies in the biology of the skin and fleece of sheep. 1 - The development and general histology of the follicle group in the skin of the Merino. Coum. Sci. Ind. Res. Aust., Bull., n" 164, 7-21.

Chase H.B., 1946. Greying induced by X-rays in the mouse. Genetics, 31, 213-214.

Chase H.B., 1954. Growth of the hair. Physiol. Rev., 34, 113-126.

Collins H.H., 1918. Studies of normal moult and artificially induced regeneration of pelage in Peromyscus. J. exp. Zool., 27, 73-99.

David L.T., 1934. Studies on the expression of genetic hairlessness in the mouse (Mus. musculus). J. exp. Zool., 68, 501-518.

Diomidova N.A., 1954. Développement embryonnaire de la peau et des poils chez le mouton. Iz. Akad. Nauk S.S.S.R., Ser. Biol., 1954, n" 6, 62-71.

DoneY J.M., Smith W.F., 1961. The fleece of the Scottish Blackface sheep. I - Seasonal changes in wool production and fleece structure. J. agric. Sci., 56, 365-374.

Dowling D.F., 1958. Seasonal changes in coat characters in cattle. Proc. Aust. Soc. anim. Prod., 2, 69-80.

Dowling D.F., 1959. The medullation characteristic of the hair coat as a factor in heat tolerance of cattle. Aust. J. agric. Res., 10, 736-743.

Downes A.M., Sharry L.F., 1971. Measurement of wool growth and its response to nutritional changes. Aust. J. biol. Sci., 24, 117-130.

DRY F.W., 1926. The coat of the mouse (Mus musculus). J. Genet., 16, 287-340.

Duerden J.E., Whitnall A.B.M., 1930. Seasonal variation in the coat of some domestic animals. S. Afr. J. Sci., 27, 521-545.

Ebling F.J., Hale P.A., 1970. The control of the mammalian moult. Mem. Soc. Endocrinol., 18, 215-238.

Ebling F.J., Johnson E., 1964. The control of hair growth. Symp. Zool. Soc. Lond., 12, 97-130.

FÉdÉRATION LAINIÈre InTERnationale, 1966. Détermination du diamètre des fibres de laine (méthode du microscope à projection), I.W.T.O-8-61, 8 pp. Réédition 1966. In : Normes adoptées par la Comm. Tech. Féd. Lain. Int., Secret. Int. Laine Ed., Londres.

Ferguson K.A., Wallace A.L.C., Linder H.C., 1965. Hormonal regulation of wool growth. In : LYNE A.G, SHORT B.F., Biology of the skin and hair growth, 655-677. Angus and Robertson, Sydney.

FLUX J.E.C., 1970. Colour change of mountain hares (Lepus timidus scoticus) in north-east Scotland. J. Zool., 162, 345-350.

Frölich G., Tegtmeyer M., 1939. Die Angora-Leistungsprüfungen. Aufgaben, Entwicklung und Ziele nach der Ergebnissen zweijähriger Versuchsarbeit auf diesem Gebiet im Tierzucht-Institut der Universität Halle. Kühn-Arch., 51, 1-68 (+3 tab.).

Haberbosch W., 1955. Untersuchungen über die Eigenschaften der Wolle des Angorakaninchens. Diss. Hohenheim, 106 pp.

Hafez E.S.E., Badreldin A.L., Shafei M.M., 1955. The hair coat in Bovinae. Emp. J. exp. Agric., 23, 34-38+ pl.

Hammond J. Jr., 1951. Control by light of reproduction in ferrets and mink. Nature, 167, 150-151.

Hammond J. Jr., 1952. Control of reproduction and pelts changes in ferrets : some experiments with animal kept entirely upon artificial light. J. agric. Sci., 42, 293-303.

HARDY M.H., LYNe A.G., 1956. The pre-natal development of wool follicles in Merino sheep. Aust. J. biol. Sci., 9, 423-441.

HarveY N.E., MCFarlane W.V., 1958. The effects of day length on the coat-shedding cycles, body-weight and reproduction on the ferret. Aust. J. biol. Sci., 11, 187-199.

Hayman R.H., 1965. Hair growth in cattle. In : Lyne A.G., ShORT B.F., Biology of the skin and hair growth, 575-590. Angus and Robertson, Sydney, $806 \mathrm{pp}$.

HaYMan R.H., NAY T., 1961. Observations on hair growth and shedding in cattle. Aust. J. agric. Sci., 12, 513-527. 
Hewson R., 1963. Moults and pelages in the brown hare Lepus europaeus occidentalis de Winton. Proc. Zool. Soc. Lond., 141, Part 4, 677-688.

InABa M., Antony J., MCKinstry C., 1979. Histologic study of the regeneration of axillary hair after removal with subcutaneous tissue shaver. J. invest. Dermatol., 72, 224-231.

Jacquemart J., Monroce R., 1965. Technique rapide de microtomie pour le laboratoire textile. Bull. Inst. Text. France, 19, 763-776.

Johnson E., 1977 a. 40 - The control of hair growth. In : JARrett A.. The physiology and pathology of the skin, 4, The hair follicle, 1351-1388. Acad. Press, London.

JoInson E., 1977 b. 41 - Environmental effects on the hair follicle. In : JARretT A., The physiology and pathology of the skin, 4, The hair follicle, 1389-1415. Acadl. Press, London.

JoHnSON E., Hornby J., 1975. Seasonal changes of pelage in the roe deer Capreolus capreolus and its role in thermoregulation. J. nat. Hist., 9, 619-628.

Kay R.N.B., Ryder M.L., 1978. Coat growth in red deer (Cervus elaphus) exposed to a day-length cycle of six months duration. J. Zool., 185, 505-510.

KETTNER B., 1962. Untersuchungen über den Einfluss von Umweltfaktoren auf Wolleistung und Wollstruktur bei Angorakaninchen verschiedener Zuchtgebiete. Kühn Arch., 76, 424-491.

Khateeb A. Al-, Johnson E., 1971 a. Seasonal changes of pelage in the vole (Microtus agrestis). I - Correlation with changes in the endocrine glands. Gen. comp. Endocrinol., 16, 217-228.

Khateeb A. Al--, Johnson E., 1971 b. Seasonal changes in pelage in the vole (Microtus agrestis). II - The effect of daylength. Gen. comp. Endocrinol., 16, 229-235.

KoETTER U., 1967. Wollerstrag, Wollqualität und Vliesbildung beim Angorakaninchen. Zïchtungskd., 69, 56-66.

Lebas F., 1975. Le lapin de chatir. Ses besoins nutritionnels et son alimentation pratique. Documents Inst. Tech. Avic. : Session Lapins. 51 pp., I.T.A.V.I., Paris.

Ling J.K., 1970. Pelage and molting in wild mammals with special reference to aquatic forms. Quartl. Rev. Biol., 45, 16-54.

LYNE A.G., 1957. The development of the epidermis and hair canals in the Merino sheep foetus. Aust, J. biol. Sci., 10, 390-397.

LyNE A.G., 1966. The development of hair follicles. Aust. I. Sci., 28, 370-377.

LYNe A.G., Brook A.H., 1964. Neogenesis of wool follicles. Aust. J. biol. Sci., 17, 514-520.

Mehner A., Koetter U., 1965. Dichte und Gruppierung der Haarfollikel der Haut des Angorakaninchens. J. Tierzücht Zïchtbiol., 81, 241-259.

MoHN M.P., 1958. The effect of different hormonal states on the growth of hair in rats. In : Montagna N., Ellis R.A., The biology of hair grow'th, 335-398. Acad. Press. New York.

Muller S.A., 1971. Hair neogenesis. J. invest. Dermatol., 56, 1-9.

Pickard J.N., 1930. Die Vererbung von Haarbüscheln und Wollmenge bei Angorakaninchen. Zï̈lttungkd., 5, 339-351.

Plitt-Hardy T.M., Dolnick E.H., 1948. Angora rabbit wool production. U.S. Dept A gric., Circ: 785,22 pp.

Priestley G.C., 1966. Rates and duration of hair growth in the albino rat. J. Anat., 100, $147-157$.

Priestley G.C., 1967. Seasonal changes in the inner root sheath of the primary follicles in Herdwick sheep. J. agric. Sci., 69, 9-12.

Rothman S., 1954. Physiology and biochemistry of the skin. Univ. Chicago Press, 741 pp.

Rothschild M., 1942. Change of pelage in the stoat, Mustela erminea L. Nature, 149, 78.

Rormschild M., 1944. Pelage change in the stoat, Mustela erminea L. Nature, 154, 180-181.

Rovgeot J., 1953. Mesure du diamètre des brins de laine. Ann. Zootech., 2, 365-376. 
Rougeot J., 1957. Action saisonnière de la durée quotidienne d'éclairement sur la mue de certaines fibres de la toison de la race ovine limousine. C.R. Soc. Biol., 51, 834-837.

Rougeot J., 1961. Action comparée des variations périodiques de la durée quotidienne d'éclairement de périodes annuelles et semestrielles sur la mue des jarres de la toison des moutons limousins. Relation avec leur cycle de reproduction. Ann. Biol. anim. Bioch. Biophys., 1, 385-402.

Rougeot J., 1972. Action du photopériodisme saisonnier sur l'activité des follicules pilcux de la toison des ovins. In : Thiers H., Cotte J., Actualités de Dermopharmacologie, vol. 4, 155-170. Centre européen de Dermopharmacologie, 33, cours Engénie, 69003 Lyon.

Rougeot J., 1974. La formation et la croissance des pelages. In : Thiers H., CotTE J., Actualités de Dermopharmacologie, vol. 6, 1-29. Centre européen de Dermopharmacologie, 33, cours Eugénie, 69003 Lyon.

Rougeot J., 1981. Les facteurs de qualité du pelage normal chez le chien. Anim. Compagnie, 16, $105-119$.

Rougeot J., Allain D., Martinet L., 1982. Photoperiodic and hormonal control of seasonal coat changes in mammals with special reference to sheep and mink. Acta zool. Finn. (sous presse).

Rougeot J., Colin M., Thebault R.G., 1980. Définition de conditions expérimentales pour l'étude des besoins nutritionnels du lapin angora : nature de la litière et présentation du lest alimentaire. Ann. Zootech., 29, 1-11.

Rougeot J., Thebault R.G., 1970. Utilisation du cyclophosphamide comme substance dépilatoire pour la récolte des poils du lapin angora. Ann. Zootech., 19, 229-234.

Rougeot J., Thebault R.G., 1976. Pratique de la récolte du poil du lapin angora par épilation après traitement au cyclophosphamide. $1^{e r}$ Cong. Int. Cunicole, Dijon, 31 mars - 2 avril 1976, Comm. n" 73, 4 pp.

Rougeot J.. Thebault R.G., Allain D., 1982. Role of the compound hair follicle in adaptative pelage changes. Acta zool. Finn. (sous presse).

Rusaovïn M., 1966. Développenent des follicules pileux chez le lapin angora. Structure et croissance du pelage de l'animal adulte. Thèse $3^{\prime}$ cycle, Fac. Sci. Univ. Paris, $59 \mathrm{pp}$.

Rudall K.M., 1955. The size and shape of the papilla in wool follicles. Proc. Int. Wool. Text. Res. Conf. Australia, F, F $9-$ F 25.

Rust C.L., Meyer R.K., 1969. Hair color, molt and testis size in male short-tailed weasels treated with melatonin. Science, 165, 921-922.

Ryder M.L., 1976. Seasonal changes in the coat of the calt. Res. vet. Sci., 21, 280-283.

Ryder M.L., 1977. Seasonal coat changes in grazing red deer (Cervus elaphus). J. Zool., 181, 137-143.

Ryder M.L., Kay R.N.B., 1973. Structure and seasonal change in the coat of red deer (Cervus elaphus). J. Zool., 170, 60-77.

Ryder M.L., Lincoln G.A., 1976. A note on the effect of changes in dialength on the seasonal wool growth cycle in Soay sheep. Anim. Prod., 23, 257-260.

Straile W.E., 1959. A study of the neoformation of mammalian hair follicles. Ann. N.Y. Acad. Sci., 83, 499-506.

Stralle W.E., 1960. Sensory hair follicles in mammalian skin : the tylotriche follicle. Am. J. Anat., 106, 133-147.

Thebault R.G., 1977. Le lapin angora. Développement post-natal de sa toison; variation saisonnière de sa production de poil. Mémoire D.P.E., Cons. Nat. Arts et Métiers, Paris, $105 \mathrm{pp}$.

Underwood L.S., ReYnolds P., 1980. Photoperiod and fur lengths in the artic fox. Int. J. Biometeor., 24, 39-48.

TrIGG M.J., 1972. Hair growth affecting coat structure. J. Zool., 168, 165-198.

WATSON A., 1963. The effect of climate in the colour changes of mountain hares in Scotland. Proc. Zool. Soc. Lond., 141, part 4, 823-835.

Whiteley H.J., 1958. Giant compound hair follicles in the skin of the rabbit. Nature, $181,850$.

Yeates N.T.M., 1955. Photoperiodicity in cattle. I - Seasonal changes in coat character and their importance in heat regulation. Aust. J. agric. Res.. 6, 891-902 +3 pl. 